\title{
Polymersomes decorated with SARS-CoV-2 spike protein receptor binding domain elicit robust humoral and cellular immunity
}

Lisa R. Volpatti ${ }^{1^{*}}$, Rachel P. Wallace ${ }^{1^{*}}$, Shijie Cao ${ }^{{ }^{*}}$, Michal M. Raczy ${ }^{1^{*}}$, Ruyi Wang ${ }^{1^{*}}$, Laura T. Gray $^{1}$, Aaron T. Alpar ${ }^{1}$, Priscilla S. Briquez ${ }^{1}$, Nikolaos Mitrousis ${ }^{1}$, Tiffany M. Marchell ${ }^{2}$, Maria Stella Sasso ${ }^{1}$, Mindy Nguyen ${ }^{1}$, Aslan Mansurov ${ }^{1}$, Erica Budina ${ }^{1}$, Ani Solanki ${ }^{3}$, Elyse A. Watkins ${ }^{1}$, Mathew R. Schnorenberg ${ }^{1}$, Andrew C. Tremain ${ }^{2}$, Joseph W. Reda ${ }^{1}$, Vlad Nicolaescu ${ }^{4}$, Kevin Furlong ${ }^{4}$, Steve Dvorkin ${ }^{4}$, Shann S. Yu ${ }^{1}$, Balaji Manicassamy ${ }^{5}$, James L. LaBelle ${ }^{6}$, Matthew V. Tirrell ${ }^{1,7}$, Glenn Randall, ${ }^{4}$ Marcin Kwissa ${ }^{1}$, Melody A. Swartz ${ }^{1,2,8,9+}$, Jeffrey A. Hubbell ${ }^{1,2,9+}$

*These authors contributed equally to this work

${ }^{1}$ Pritzker School of Molecular Engineering, University of Chicago, Chicago, IL 60637, United States

${ }^{2}$ Committee on Immunology, University of Chicago, Chicago, IL 60637, United States

${ }^{3}$ Animal Resources Center, University of Chicago, Chicago, IL 60637, United States

${ }^{4}$ Department of Microbiology, Howard T. Ricketts Laboratory, University of Chicago, Chicago, IL 60637, United States

${ }^{5}$ Department of Microbiology and Immunology, University of lowa, lowa City, IA 52242, United States

${ }^{6}$ Department of Pediatrics, University of Chicago Comer Children's Hospital, Chicago, IL 60637, United States

${ }^{7}$ Materials Science Division, Argonne National Laboratory, Lemont, IL 60439, United States

${ }^{8}$ Ben May Department of Cancer Research, University of Chicago, Chicago, IL 60637, United States

${ }^{9}$ Committee on Cancer Biology, University of Chicago, Chicago, IL 60637, United States

+Correspondence to melodyswartz@uchicago.edu, jhubbell@uchicago.edu 


\section{ABSTRACT}

A diverse portfolio of SARS-CoV-2 vaccine candidates is needed to combat the evolving COVID19 pandemic. Here, we developed a subunit nanovaccine by conjugating SARS-CoV-2 Spike protein receptor binding domain (RBD) to the surface of oxidation-sensitive polymersomes. We evaluated the humoral and cellular responses of mice immunized with these surface-decorated polymersomes ( $\left.\mathrm{RBD}_{\text {surf }}\right)$ compared to RBD-encapsulated polymersomes ( $\left.\mathrm{RBD}_{\text {encap }}\right)$ and

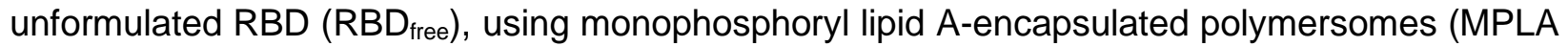
PS) as an adjuvant. While all three groups produced high titers of RBD-specific IgG, only $R B D_{\text {surf }}$ elicited a neutralizing antibody response to SARS-CoV-2 comparable to that of human convalescent plasma. Moreover, $\mathrm{RBD}_{\text {surf }}$ was the only group to significantly increase the proportion of RBD-specific germinal center $B$ cells in the vaccination-site draining lymph nodes. Both $\mathrm{RBD}_{\text {surf }}$ and $\mathrm{RBD}_{\text {encap }}$ drove similarly robust $\mathrm{CD} 4^{+}$and $\mathrm{CD} 8^{+} \mathrm{T}$ cell responses that produced multiple Th1-type cytokines. We conclude that multivalent surface display of Spike RBD on polymersomes promotes a potent neutralizing antibody response to SARS-CoV-2, while both antigen formulations promote robust $\mathrm{T}$ cell immunity.

\section{INTRODUCTION}

COVID-19, the disease caused by the novel coronavirus SARS-CoV-2, emerged in late 2019 and was declared a pandemic by the World Health Organization in March 2020. Since its emergence, researchers across the world have sought to rapidly develop vaccine candidates, some of which have received Emergency Use Authorization by the U.S. Food and Drug Administration ${ }^{1,2}$. While the first vaccines that entered the clinic were based on nucleic acid technologies, subunit vaccines are gaining attention and have also shown promise in clinical trials ${ }^{3,4}$. The primary antigens used in preclinical and clinical vaccine candidates are the Spike protein and its constituent receptorbinding domain (RBD). The RBD of the Spike protein binds to the ACE-2 receptor on host cell surfaces, enabling viral entry into the host cell5,6.

Several highly potent neutralizing antibodies have been isolated that target RBD and prevent viral binding and uptake, making it an attractive vaccine target ${ }^{7-10}$. Since RBD is smaller ( 25 kDa) and more stable than the full homotrimeric Spike fusion protein ( 180 kDa), it is also advantageous from a manufacturing and distribution perspective ${ }^{11}$. However, RBD has been shown to have lower immunogenicity than the full Spike protein or its RBD-containing S1 domain ${ }^{12,13}$. Materials science and engineering approaches, particularly strategies involving nanotechnology, may improve RBD immunogenicity and thus aid in the development of nextgeneration vaccines ${ }^{14-16}$. Indeed, several approaches of self-assembling RBD into virus-like particles have resulted in potent neutralizing antibody responses ${ }^{17-20}$.

In order to offer robust protection from infection, cellular in addition to humoral responses are needed $^{21-23}$. Almost all convalescent individuals show $\mathrm{T}$ cell immunity, and the majority have both $\mathrm{CD}^{+}$and $\mathrm{CD}^{+}$SARS-CoV-2-specific T cells ${ }^{24-27}$. Conversely, severe disease is associated with lymphopenia and reduced T cell function ${ }^{28-30}$. Furthermore, T cell immunity may be more durable than humoral responses, and $\mathrm{T}$ cells are expected to play an important role in immune memory $23,28,31$. Therefore, the goal of this study was to improve both humoral and cellular immunogenicity of RBD and compare the efficacy of engineered nanoparticle formulations in order to inform the design of next-generation nanovaccines. 
We have previously reported the development of polymersomes (PS) that self-assemble from the oxidation-responsive block copolymer poly(ethylene glycol)-bl-poly(propylene sulfide) (PEGPPS $)^{32}$ and shown their efficacy in delivering antigen and adjuvant to dendritic cell endosomes ${ }^{33}$. In endolysosomal compartments, the PPS block becomes oxidized, which initiates the restructuring of the PS into micelles and concurrent release of encapsulated payload ${ }^{33,34}$. These vaccine nanocarriers have been shown to activate dendritic cells, induce robust $T$ cell immunity, and elicit high antibody titers with broad epitope coverage ${ }^{33,35,36}$.

In this study, we hypothesized that we could further improve the humoral responses elicited by PS while retaining their ability to induce $T$ cell immunity by engineering them to mimic the physical form of a viral particle through multivalent surface display of antigen. We envisaged that multivalent surface display of RBD would result in enhanced crosslinking and clustering of B cell receptors (BCRs) and subsequent production of neutralizing antibodies. Here, we report on the development and preclinical evaluation of PS displaying surface-bound RBD (RBD surf) and PS encapsulating RBD (RBD encap) adjuvanted with monophosphoryl lipid A-encapsulated PS (MPLA $P S)$. We show that mice vaccinated with $R_{B D}$ surf in combination with MPLA PS in a prime-boost schedule develop high titers of SARS-CoV-2-neutralizing antibodies with robust germinal center responses as well as $\mathrm{CD}^{+}$and $\mathrm{CD}^{+} \mathrm{T}$ cell immunity, thus meeting our design criteria.

\section{RESULTS}

\section{Formulated polymersomes exhibit long-term stability and in vitro activity}

Having previously encapsulated antigen into PS as nanovaccines ${ }^{33}$, here we developed a conjugation strategy to attach antigens to their surface. To create a modular platform that could be generalized to any antigen, we synthesized $\mathrm{N}_{3}$-PEG-PPS (Suppl. Fig. S1), which, when formulated into PS, yields particles displaying clickable surface moieties (Fig. 1a). Upon the addition of RBD conjugated to a DBCO-containing linker, we generated PS decorated with

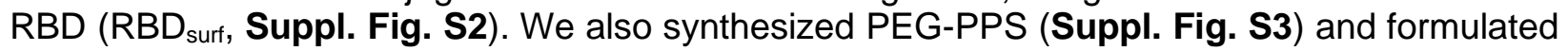

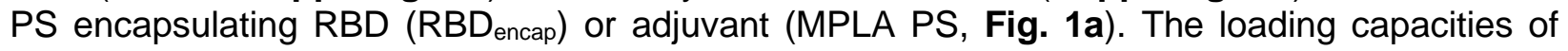
$\mathrm{RBD}_{\text {surf }}$ and $\mathrm{RBD}_{\text {encap }}$ were $1.57 \%$ and $1.75 \%$, respectively, comparable to previous reports of encapsulated ovalbumin ${ }^{33,35}$, while the loading capacity of MPLA PS was $6.46 \%$ (Suppl. Table S1). We confirmed the vesicular structure of PS through cryo-electron microscopy (cryoEM) and demonstrated that the different formulations have similar sizes and morphologies (Fig. 1b, Suppl. Fig. S4). According to dynamic light scattering (DLS) measurements, the average PS diameter is around $150 \mathrm{~nm}$ (Fig. 1C,d), which is similar to the reported size of SARS-CoV-2 particles (60-140 $\mathrm{nm})^{37}$.The polydispersity index (PDI) of each formulation was $<0.2$, indicative of a relatively homogenous population of nanoparticles. As indicated by their consistent size and PDI, in addition to the absence of free RBD released into solution, PS remain stable at $4{ }^{\circ} \mathrm{C}$ for at least 180 days, which can be beneficial for distribution and shelf-life considerations (Fig. 1c, Suppl. Fig. S5).

We next characterized the biological activity of the PS formulations in vitro. To confirm that RBD structure is not substantially altered upon conjugation to the PS surface, we quantified its ability to bind to HEK-293 cells that express human ACE-2 (HEK-hACE2, Fig. 1e). The normalized mean fluorescence intensity (MFI) versus RBD concentration curves were used to calculate the equilibrium dissociation constants $\left(K_{D}\right)$ for free $R B D$ and $R B D_{\text {surf }}$ conjugated to AF647 (AF647$R B D_{\text {free }}$ and $A F 647-R B D_{\text {surf, }}$ respectively). The curves and $K_{D}$ values are in excellent agreement, indicating that surface conjugation to PS did not impact ACE-2 binding of RBD. Empty PS conjugated to AF647 did not bind to HEK-hACE2, and neither PS formulation bound to HEK-293 
cells lacking hACE-2 (Suppl. Fig. S6). Next, we confirmed that MPLA retained its ability to serve as a TLR4 agonist upon formulation in PS with a HEK-Blue ${ }^{\text {TM }}$ TLR4 reporter cell line (Suppl. Fig. S7). To further validate MPLA PS activity in a more physiologically-relevant model, we stimulated murine bone marrow-derived dendritic cells (BMDCs) with free MPLA, MPLA PS, or empty PS, and we measured the subsequent secretion of the pro-inflammatory cytokines TNFa, IL-6, IL-1a, and IL-1 $\beta$ (Fig. 1f, Suppl. Fig. S8). For each cytokine, there was a dose-dependent increase in secretion for free MPLA and MPLA PS with only background levels of secretion for empty PS, indicating that MPLA PS successfully activated antigen presenting cells (APCs) in vitro. Thus, we successfully synthesized two RBD formulations of PS in addition to MPLA PS and showed that they are homogenous vesicular structures with long-term stability and in vitro biological activity.
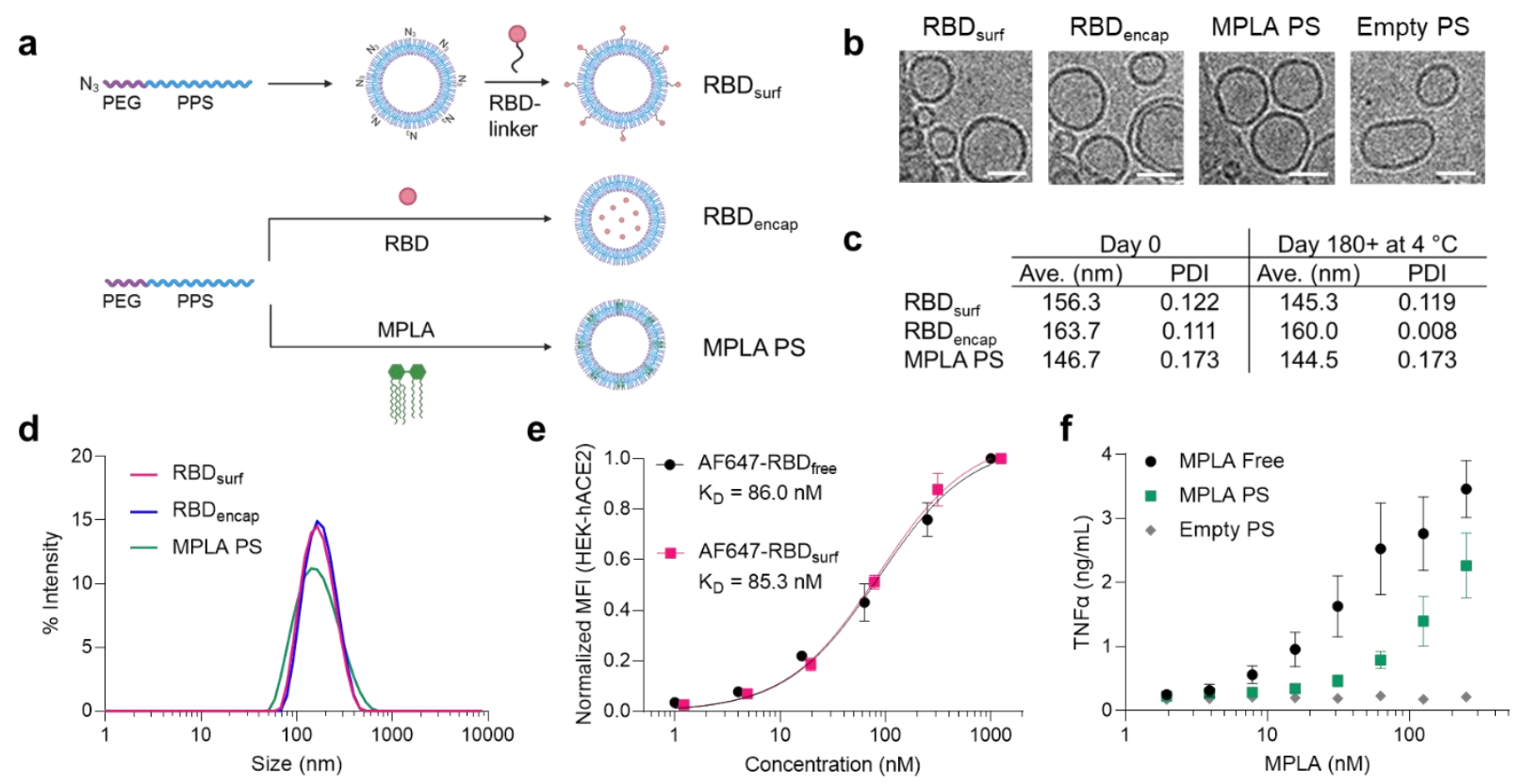

Figure 1 | RBD and MPLA are formulated into stable, biologically active polymersomes (PS). a, Schematic of formulation of PS. RBD was conjugated to the surface $\left(\mathrm{RBD}_{\text {surf }}\right)$ or encapsulated inside $\left(\mathrm{RBD}_{\text {encap }}\right)$ of PS, and MPLA was encapsulated in the vesicle membrane (MPLA PS) due to its hydrophobicity. b, Representative cryo-electron microscopy images of PS, depicting vesicle structure. Scale $=50 \mathrm{~nm}$. c, Size and polydispersity index (PDI) from dynamic light scattering (DLS) measurements of PS upon formulation and after $>6$ months at $4{ }^{\circ} \mathrm{C}$. d, Representative DLS curves of PS. e, Normalized mean fluorescence intensity (MFI) of AF647 conjugated to free RBD or RBD surt by flow cytometry showing concentration-dependent binding to HEK-293 cells that express human ACE-2 (HEK-hACE2). Nonlinear regression was used to model data assuming specific binding to one site to determine equilibrium dissociation constants. f, Dose-dependent secretion of TNFa from cultured murine bone marrow-derived dendritic cells (BMDCs) stimulated by free MPLA, MPLA PS, or empty PS. Data represent mean \pm SD for $n=2$ (e) or 3 (f) replicates. 


\section{All adjuvanted formulations elicit RBD-specific IgG responses}

Having confirmed that antigen- and adjuvant-loaded PS exhibit their expected bioactivity in vitro, we next evaluated their ability to enhance humoral and cellular immunity in mice compared to $\mathrm{RBD}_{\text {free. }}$ We immunized mice via s.c. injection in the hocks in a prime-boost schedule 3 weeks apart and monitored antibody titers weekly (Fig. 2a). The total RBD-specific IgG is represented by the area under the log-transformed ELISA absorbance curves (AUC), starting at a plasma dilution of $10^{-2}$ (see Methods, Suppl. Fig. S9). All adjuvanted groups had significant anti-RBD binding antibody responses within a week after their first dose, with $\mathrm{RBD}_{\text {encap }}$ stimulating the highest responses (Fig. 2b). The antibody responses in adjuvanted groups either increased gradually or remained constant until a week after the booster, when the mean AUC increased 1.3- to 1.6-fold.

In order to explore the humoral response in further detail, we then evaluated IgG subtypes of induced antibodies at the study endpoint (d28, 1 week post-boost). While plasma antibody levels of all adjuvanted groups were similar for $\lg G 1$ and $\lg G 3, R_{B D}$ surf elicited significantly lower lgG2b and IgG2c antibody responses (Fig. 2c). The ratio of IgG2b/lgG1 was then taken as an indication of a Th1/Th2-mediated response ${ }^{38}$. While $R B D_{\text {encap }}$ and $R B D_{\text {free }}+$ MPLA PS have a ratio of around 1 , indicating a balanced Th1/Th2 response, $R B D_{\text {surf }}$ shows a lower ratio of lgG2b to IgG1 indicating a slightly Th2-skewed response (Fig. 2d). Since $\lg A$ is important for combating respiratory viruses at the mucosal sites, we also measured plasma levels of RBD-specific $\lg A$ antibodies $^{39,40}$. Although we did not expect high titers of circulating IgA from a s.c. administered vaccine, there were detectable anti-RBD IgA antibodies in all adjuvanted groups, which were significantly higher than the background-level responses elicited by unadjuvanted RBD free (Suppl. Fig. S10).

Next, to determine if the higher antibody responses of adjuvanted groups stemmed from an expanded number of RBD-specific antibody secreting cells (ASCs), we performed an ex vivo RBD enzyme-linked immunosorbent spot (ELISpot) assay with splenocytes harvested 1 week postboost. All groups receiving adjuvanted RBD showed significantly higher RBD-specific IgG+ ASCs compared to unadjuvanted $\mathrm{RBD}_{\text {free, }}$, consistent with plasma antibody levels (Fig. 2e,f).

Finally, we evaluated the kinetics and durability of the humoral response to demonstrate the persistence of elicited antibodies (Fig. 2g). The RBD-specific IgG AUC for all adjuvanted groups increased until 1 week post-boost and then remained constant over the subsequent 4 weeks, indicating that the antibody responses stimulated by these vaccine formulations persist for at least 2 months in mice after the initial dose. Taken together, MPLA PS-adjuvanted RBD surf, $R B D_{\text {encap, }}$ and $\mathrm{RBD}_{\text {free }}$ all stimulated persistent anti-RBD antibodies and increased the frequencies of RBDspecific ASCs in the spleen. 
a

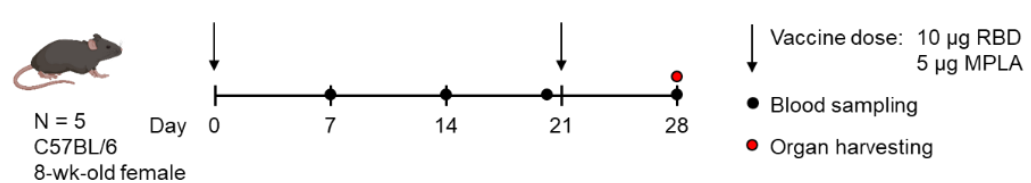

b
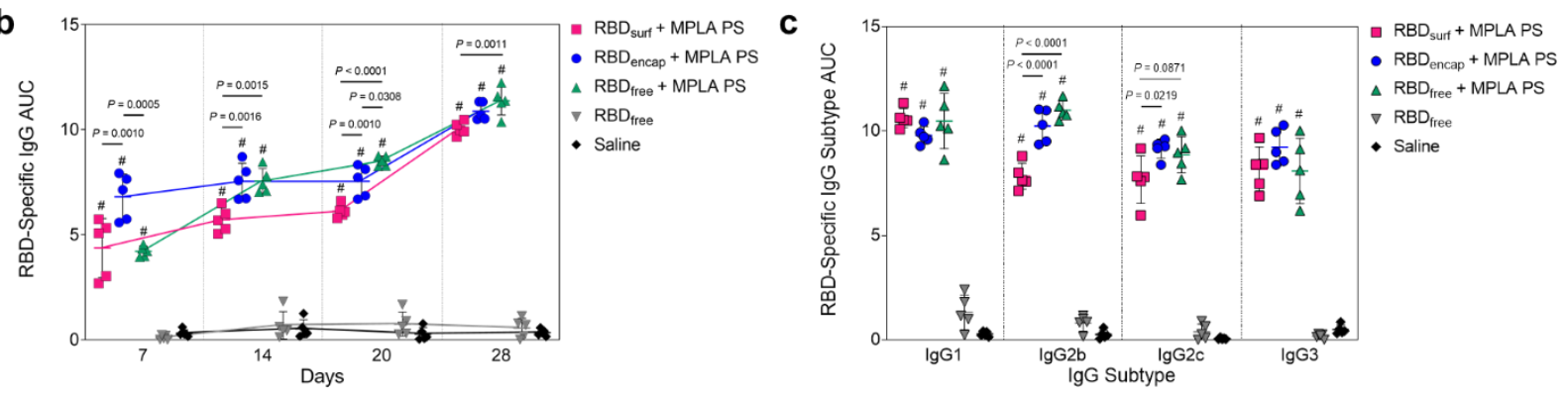

d

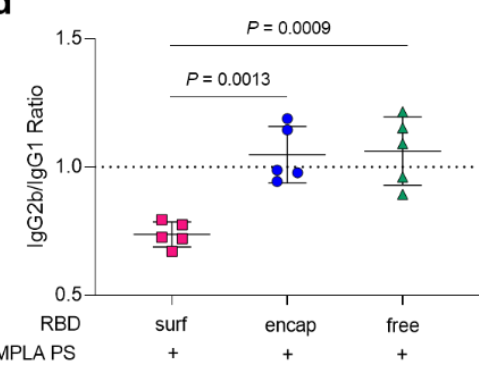

e

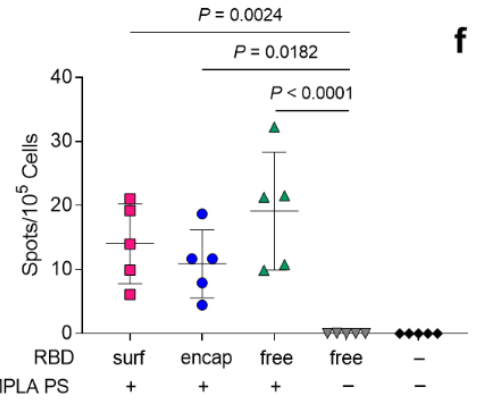

f

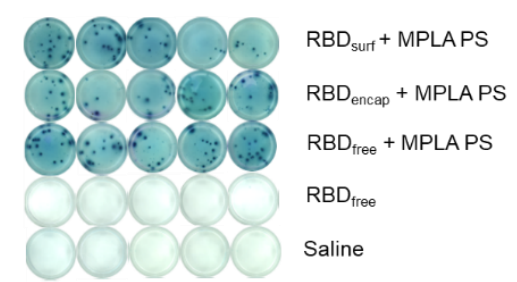

g

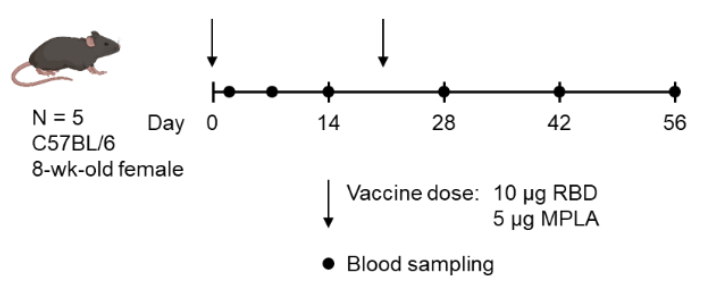

h

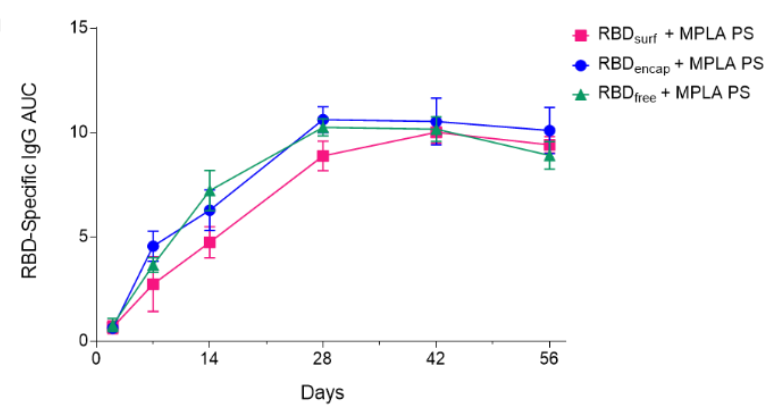

Figure 2 | High levels of RBD-specific IgG antibodies are produced upon PS vaccination. a, Vaccination schedule consisting of a priming dose followed by a booster 3 weeks later. $\mathbf{b}$, Total RBD-specific IgG antibodies over time reported as the area under the log-transformed curve (AUC) of absorbance vs. dilution. c, Comparison of RBD-specific IgG isotypes (IgG1, IgG2b, IgG2c, IgG3) on day 28. d, Ratio of AUCs of IgG2b:IgG1 isotypes. e, Quantification of RBDspecific IgG+ antibody secreting cells by ELISpot of splenocytes (Dunn's post-test compared to unadjuvanted $\mathrm{RBD}_{\text {free }}$ ). $\mathbf{f}$, Representative ELISpot wells from (e). Data plotted as mean $\pm \mathrm{SD}$ and represent 1 of 2 experiments with $n=5$ mice each. Symbols represent individual mice. g, Vaccine and blood sampling schedule of long-term kinetics study. $\mathbf{h}$, Total RBD-specific IgG antibodies over time for the vaccination schedule in (g). Data represent mean \pm SD for $n=5$ mice. Comparisons were made using one-way ANOVA with Tukey's post-test unless stated otherwise. \# $P<0.0001$ compared to unadjuvanted $\mathrm{RBD}_{\text {iree }}$. 


\section{RBD-surface-decorated polymersomes, but not RBD-encapsulated polymersomes, induce neutralizing antibodies}

After analyzing the quantity of RBD-specific antibodies produced by the vaccine candidates, we next sought to determine their neutralizing capacity and breadth of epitope recognition. Neutralizing antibodies were assessed against SARS-CoV-2 infection of Vero E6 cells in vitro. Although all adjuvanted groups elicited similarly high titers of RBD-binding IgG antibodies (105$10^{7}$, Suppl. Fig. S11a), only $\mathrm{RBD}_{\text {surf }}$ neutralized the virus to a greater extent than unadjuvanted $\mathrm{RBD}_{\text {free }}$ at a plasma dilution of $10^{-2.11}$ (Fig. 3a). We then quantified the viral neutralization titer (VNT) as the dilution at which $50 \%$ of SARS-CoV-2-mediated cell death is neutralized. There was no significant difference between VNTs of human convalescent individual samples and $\mathrm{RBD}_{\text {surf }}$ plasma, and both groups induced higher VNTs compared to unadjuvanted $R_{B D}$ free (Fig. 3b). Furthermore, the median VNT elicited by RBD surf was 2.45, which falls within the FDA classification of "high titer" for convalescent plasma therapy $(>2.40)^{41}$. To ensure reproducibility, the neutralization assay was repeated with 3 different cohorts of $n=5$ mice each, and no significant differences were observed between the resulting VNTs (Suppl. Fig. S11b).

We next aimed to determine whether differences in neutralizing ability resulted from the epitope recognition breadth elicited by each vaccine formulation. To test this, we mapped the epitopes recognized by vaccine-elicited antibodies using a linear peptide array from the full-length $R B D$ sequence. While $\lg G$ antibodies elicited by $R B D_{\text {surf }}$ primarily recognized linear epitopes concentrated within the receptor binding motif of RBD (RBM; aa $438-508$ ), $R B D_{\text {encap }}$ and $R B D_{\text {free }}$ + MPLA PS exhibited broader linear epitope diversity (Fig. 3c,d, Suppl. Fig. S12). Within RBD, the RBM is particularly critical for interacting with ACE-2, so antibodies specific for this region may have potent neutralizing potential ${ }^{42,43}$.

Because $R B D_{\text {surf }}$ appeared to offer the advantage of improved neutralizing activity, while $R B D_{\text {encap }}$ offered epitope diversity, we asked if co-administration would synergize to further enhance protection. To test this, we mixed $R B D_{\text {surf }}$ and $R B D_{\text {encap }}$ (with total $R B D$ dose remaining constant) with MPLA PS and treated mice using the same vaccination schedule. As an additional control, we also investigated the humoral response of $R B D_{\text {free }}$ adjuvanted with free MPLA. While both groups produced high RBD-specific IgG AUCs, neither exhibited neutralizing potential against the SARS-CoV-2 virus above background levels (Suppl. Fig. S13a,b). Analysis of the peptide arrays for these groups shows the presence of high-intensity-binding antibodies outside of the RBM (Suppl. Fig. S13c).

In summary, while all adjuvanted groups elicit high titers of RBD-specific antibodies, only RBD surf generated neutralizing antibodies against SARS-CoV-2 at titers comparable to human convalescent plasma. Additionally, these antibodies uniquely bound to linear epitopes localized within the RBM, while all other groups produced antibodies with greater epitope breadth. 
a
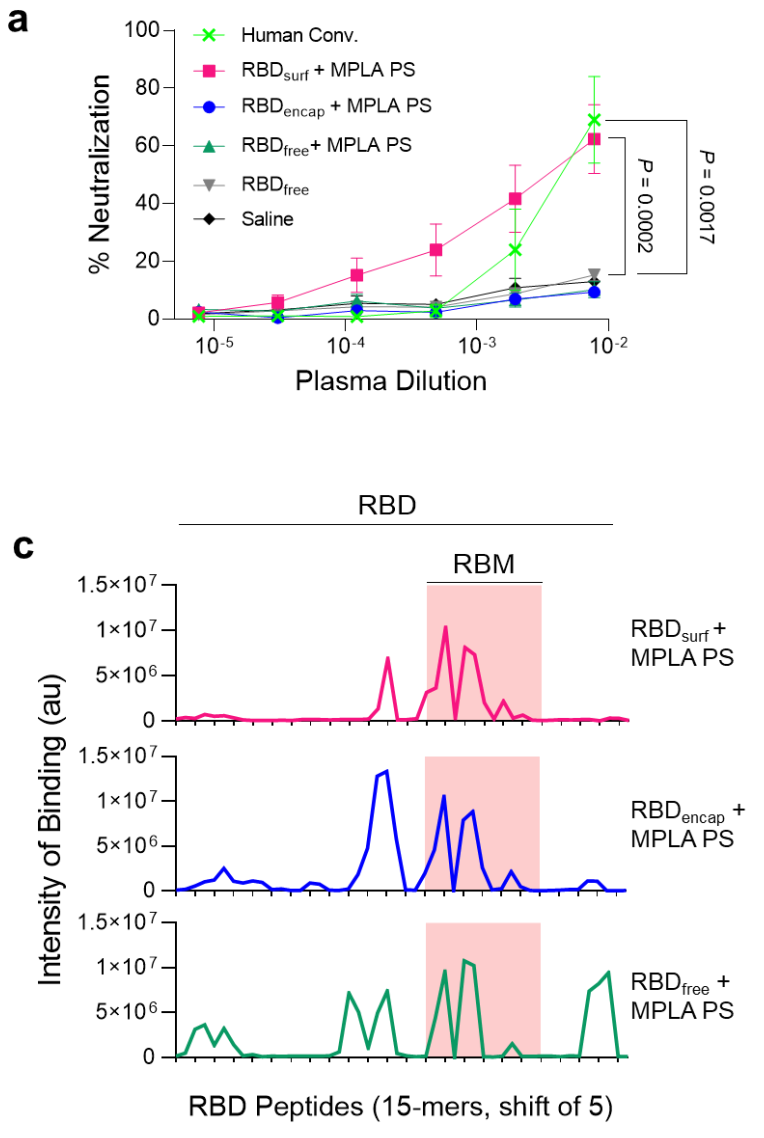

b

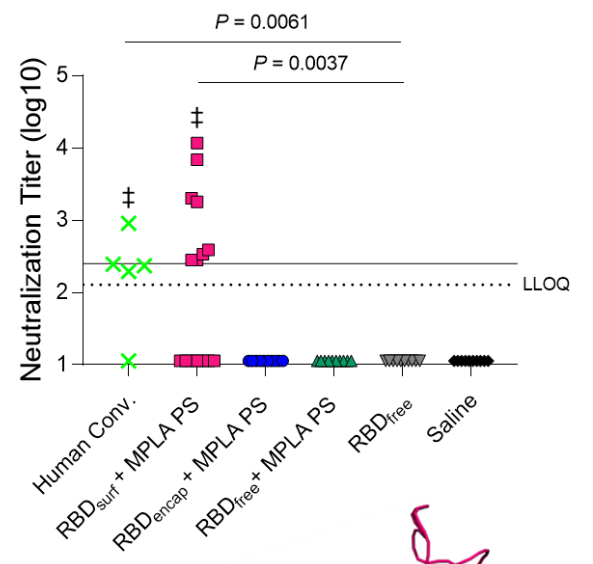

Figure 3 | Antibodies induced by vaccination with RBD surf $_{+}$MPLA PS are neutralizing and localized to the receptor binding motif. a, Plasma from mice 1 week post-boost was tested for its ability to neutralize SARS-CoV-2 infection of Vero E6 cells in vitro. Percent neutralization for multiple plasma dilutions normalized to cells without virus $(100 \%)$ or without plasma $(0 \%)$. Data plotted as mean \pm SEM for $n=5$ convalescent human samples (human conv.) or 10-15 mice. Comparisons to unadjuvanted $\mathrm{RBD}_{\text {free }}$ were made for lowest dilution $\left(10^{-2.11}\right)$ using one-way ANOVA with Dunnett's post-test. $\mathbf{b}$, Viral neutralization titers, representing the plasma dilution at which $50 \%$ of SARS-CoV-2-mediated cell death is neutralized. Dashed line: lower limit of quantification $(L L O Q=2.11)$. For values below the $L L O Q, L L O Q / 2$ values were plotted. Solid line: FDA recommendation for "high titer" classification $(=2.40)$. Comparisons were made using Kruskal-Wallis nonparametric test with Dunn's post-test or Wilcoxon signed rank test $\left(^{\ddagger} \mathrm{ns}, P>\right.$ 0.05 compared to hypothetical value of 2.40 ). Symbols represent individual mice. c, Pooled plasma was then tested for antibody binding to linear epitopes using overlapping 15-amino-acid peptides with 5-amino-acid offsets, spanning the entire RBD sequence. X-axis represents sequential peptide number within the RBD amino acid sequence, and y-axis represents average luminescence for each peptide epitope. $\mathbf{d}$, 3D structure of RBD with the receptor binding motif (RBM) underlined and main peptide sequences recognized by mouse plasma highlighted in pink (Protein Data Bank Entry 7DDD). 


\section{All adjuvanted formulations increase $T_{\text {fh }}$ and $B$ cell activation in the dLN}

Given the differences in antibody responses and neutralizing activity elicited by $R B D_{\text {surf }}$ versus $\mathrm{RBD}_{\text {encap }}$ vaccination, we further investigated the phenotypes of the $\mathrm{B}$ and $\mathrm{T}$ cells involved in the initiation of the humoral immune response. All adjuvanted groups showed trends of higher frequencies of $\mathrm{T}$ follicular helper cells $\left(\mathrm{T}_{\mathrm{fh}}\right.$; $\left.\mathrm{CD} 4^{+} \mathrm{CXCR} 5^{+} \mathrm{BCL} 6^{+}\right)$in the injection site-draining lymph nodes (dLNs) 1 week post-boost compared to unadjuvanted RBD free (Fig. 4a, Suppl. Fig. S14), although differences were only statistically significant for RBD free + MPLA PS. Interestingly, a greater percentage of $T_{\text {fh }}$ cells in all adjuvanted groups highly upregulated expression of ICOS, a co-stimulatory receptor important in $\mathrm{T}_{\text {fh }}$ activation and maintenance (Fig. $\left.\mathbf{4 b}\right)^{44}$.

Following activation by $\mathrm{T}_{\text {fh }}$ cells, naïve $\mathrm{B}$ cells can either undergo a germinal center (GC)dependent response in which they become GC B cells and undergo cycles of class-switching and somatic hypermutation (SHM) before differentiation into long-lived plasma cells and classswitched memory B cells, or they can differentiate into short-lived plasmablasts or IgM memory cells in a GC-independent response ${ }^{45}$. A stronger GC response is valuable in vaccination because it results in higher affinity and longer-lived antibody production ${ }^{46}$. Overall frequencies of $B$ cells $\left(\mathrm{CD} 19^{+} \mathrm{B}^{2} 20^{+}\right)$were unaffected across groups, but there were significantly lower frequencies of

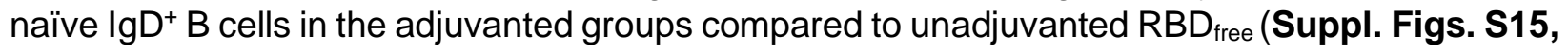
S16). All adjuvanted groups generated GC responses, characterized by increased frequencies of GC B cells (CD38-GL7+) in the dLN compared to unadjuvanted $R B D_{\text {free }}$ (Fig. 4c). Both $R B D_{\text {encap }}$

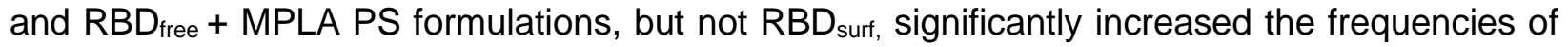
plasmablasts $\left(B 220^{+} \mathrm{CD}_{138^{+}}\right)$in the dLN compared to unadjuvanted $\mathrm{RBD}_{\text {free }}$ (Fig. 4d).

To determine the antigen-specificity of the B cells, we developed a set of fluorescent RBD protein tetrameric probes. To ensure selectivity, B cells were considered RBD-specific if they were double-positive for both PE- and APC-conjugated RBD-tetramers and negative for the nonspecific control protein tetramer (Suppl. Fig. S17). RBD free + MPLA PS was the only formulation to significantly increase the frequency of RBD-specific B cells in the dLN (Suppl. Fig. S18). We next sought to further determine the phenotype of these RBD-specific $B$ cells. $R B D_{\text {surf }}$, unlike the other adjuvanted formulations, led to a significantly higher fraction of RBD-specific B cells with GC B cell phenotype, suggesting a more robust $G C$ response to $R B D$ (Fig. 4 e). $R B D_{\text {surf }}$ was also the only formulation with a significantly lower fraction of plasmablasts within the RBD-specific $B$ cell subset compared to unadjuvanted $R B D_{\text {free }}$ (Fig. 4f). These differences are also visually apparent in pooled flow cytometry plots for RBD-specific GC B cells (Fig. $\mathbf{4 g}$ ) and plasmablasts (Fig. 4h). In summary, all adjuvanted formulations of RBD increased activation of $T_{\text {fh }}$ cells and GC B cells in the dLN, but within the RBD-specific B cell population, only RBD surf generated a higher fraction of GC B cells and a lower fraction of plasmablasts. 

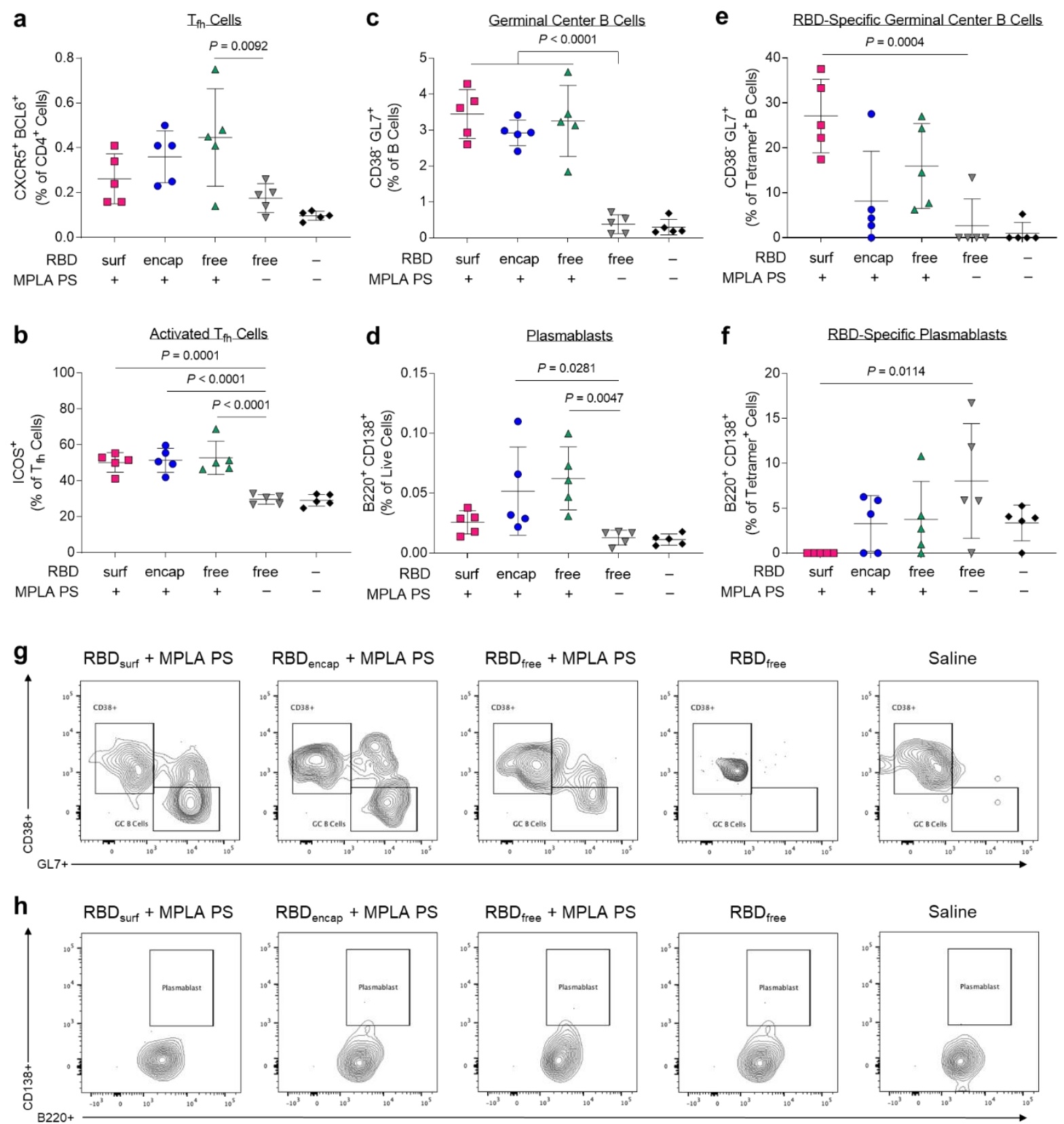

Figure $4 \mid \mathrm{CD}^{+}{ }^{+} \mathrm{T}$ follicular helper cell $\left(\mathrm{T}_{\mathrm{fh}}\right)$ and $\mathrm{B}$ cells are activated by PS vaccine 1 week post-boost in the injection site-draining lymph nodes (dLN). a, $\mathrm{T}_{\text {fh }}$ cells (CXCR5 ${ }^{+} \mathrm{BCL} 6^{+}$) of vaccinated mice quantified via flow cytometry as a percent of live $C D 4^{+} T$ cells. $\mathbf{b}$, Highly activated ICOS $^{+} T_{\text {th }}$ cells quantified as percent of $T_{\text {fh }}$ cells. c, Germinal center B cells (CD38- GL7+) quantified as a percentage of total B cells $\left(B 220^{+} \mathrm{CD} 19^{+}\right)$. d, Plasmablasts (B220+ CD138+) quantified as a percentage of total dLN cells. e, Germinal center $B$ cells quantified as a percentage of RBD-specific B cells. f, Plasmablasts quantified as a percentage of RBD-specific dLN cells. g$h$, Concatenated flow cytometry contour plots for $n=5$ mice/group showing RBD-specific GC B cells $(\mathrm{g})$ or plasmablasts $(\mathrm{h})$. Data plotted as mean $\pm \mathrm{SD}$ and represent 1 of 2 experiments with $\mathrm{n}$ $=5$ mice each. Symbols represent individual mice. Comparisons to unadjuvanted $\mathrm{RBD}_{\text {free }}$ were made using one-way ANOVA with Dunn's post-test. 


\section{Vaccination with polymersome-formulated RBD generates RBD-specific Th1 T cell responses}

Having demonstrated that our PS vaccines can generate strong humoral responses, we next sought to determine their capacity to generate robust $\mathrm{CD} 8^{+}$and $\mathrm{CD} 4^{+} \mathrm{T}$ cell immunity. In order to assess the RBD-specific T cell response, we isolated cells from the dLNs of vaccinated mice 1 week post-boost. Prior to intracellular staining, cells were restimulated with RBD peptide pools for 6 hours. The RBD-specific response was quantified by subtracting the signal from cells incubated in media alone from those incubated with RBD peptide pools (Suppl. Fig. S19). Only PSformulated RBD groups $R B D_{\text {surf }}$ and $R B D_{\text {encap }}$ generated significantly higher frequencies of IFN $\gamma^{+}$, bifunctional IFN $\gamma^{+} \mathrm{TNF} \alpha^{+}$, and IL-2 $2^{+}$secreting $\mathrm{CD}^{+} \mathrm{T}$ cells compared to unadjuvanted $\mathrm{RBD}_{\text {free }}$ (Fig. 5a). Similar trends were seen in the CD4 ${ }^{+} \mathrm{T}$ cell compartment. Treatment with $\mathrm{RBD}_{\text {surf }}$ and $\mathrm{RBD}_{\text {encap }}$ but not $\mathrm{RBD}_{\text {free }}+$ MPLA PS led to significantly higher frequencies of IFN $\gamma^{+}$and IL-2 $2^{+}$ secreting $\mathrm{CD}^{+} \mathrm{T}$ cells, although the increase in bifunctional IFN $\gamma^{+} \mathrm{TNF} \alpha^{+}$was not statistically significant (Fig. 5b). The production of these cytokines implies a Th1 T cell response, which is correlated with less severe cases of SARS-CoV infection ${ }^{21}$.

For further validation of the cytokine response, cells from the dLN isolated from the same vaccinated mice were restimulated with full RBD protein ex vivo for 3 days, followed by quantification of cytokines in the supernatant. The RBD-specific response was quantified by subtracting unstimulated signal from stimulated signal as above. The levels of Th1-type cytokines detected were consistent with intracellular staining data. Levels of IFN $\gamma$ and IL-2 were modestly but not significantly increased in the $R B D_{\text {surf }}$ and $R B D_{\text {encap }}$ groups compared to the $R B D_{\text {free }}$ group, while cells from $\mathrm{RBD}_{\text {encap }}$-treated mice produced TNF $\alpha$ at significantly higher levels than unadjuvanted $R B D_{\text {free }}$ (Fig. 5c). RBD encap also led to increased production of IL-6 and IL-10, which are pleiotropic cytokines known to be secreted during Th1 responses (Fig. 5c ${ }^{47,48}$. Levels of secreted Th2-type cytokines were also measured. More IL-4 was produced in the supernatant of samples treated with $\mathrm{RBD}_{\text {surf }}$ and $\mathrm{RBD}_{\text {encap }}$ compared to $\mathrm{RBD}_{\text {free }}$, albeit at an overall low concentration. There was no significant elevation of IL-5 secretion across any of the treatment groups compared to the saline control and no detectable levels of IL-13 in any sample (Suppl. Fig. S20). In summary, vaccination with RBD delivered via polymersome formulations generated stronger RBD-specific Th1-type $\mathrm{CD}^{+}$and $\mathrm{CD}^{+} \mathrm{T}$ cell responses than unadjuvanted $\mathrm{RBD}_{\text {free, }}$ while RBD free + MPLA PS did not. 


\section{$\underline{6 \mathrm{~h} \text { ex vivo peptide pool restimulation }}$}
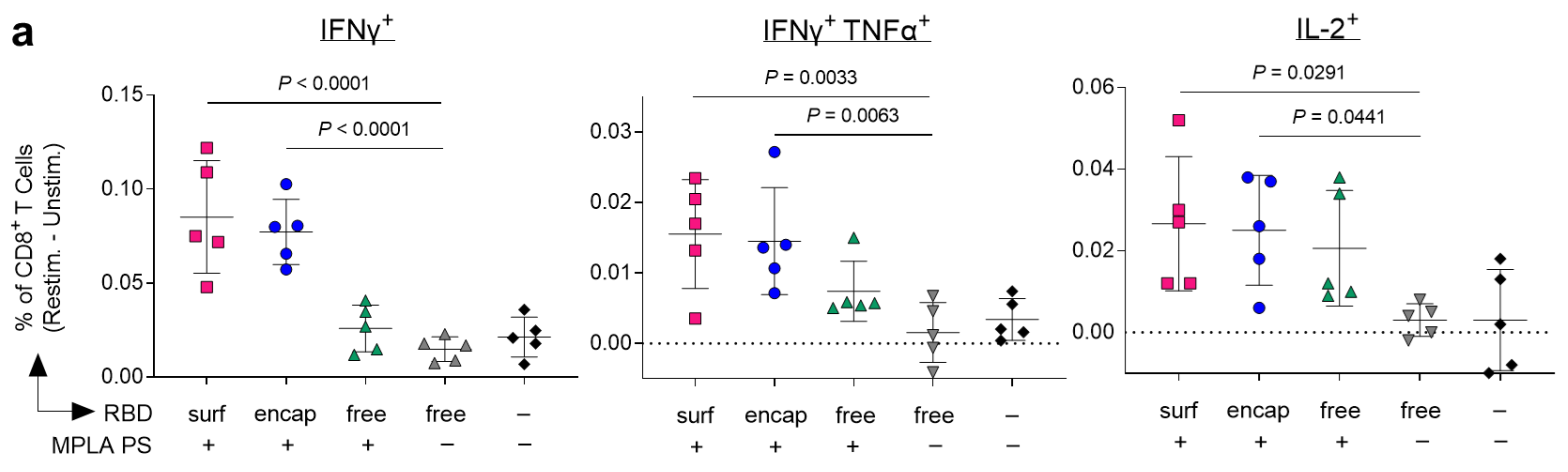

b

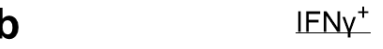

$\underline{\mathrm{FNy}^{+} \mathrm{TNFa}^{+}}$

$\mathrm{LL}^{+} \mathbf{2}^{+}$
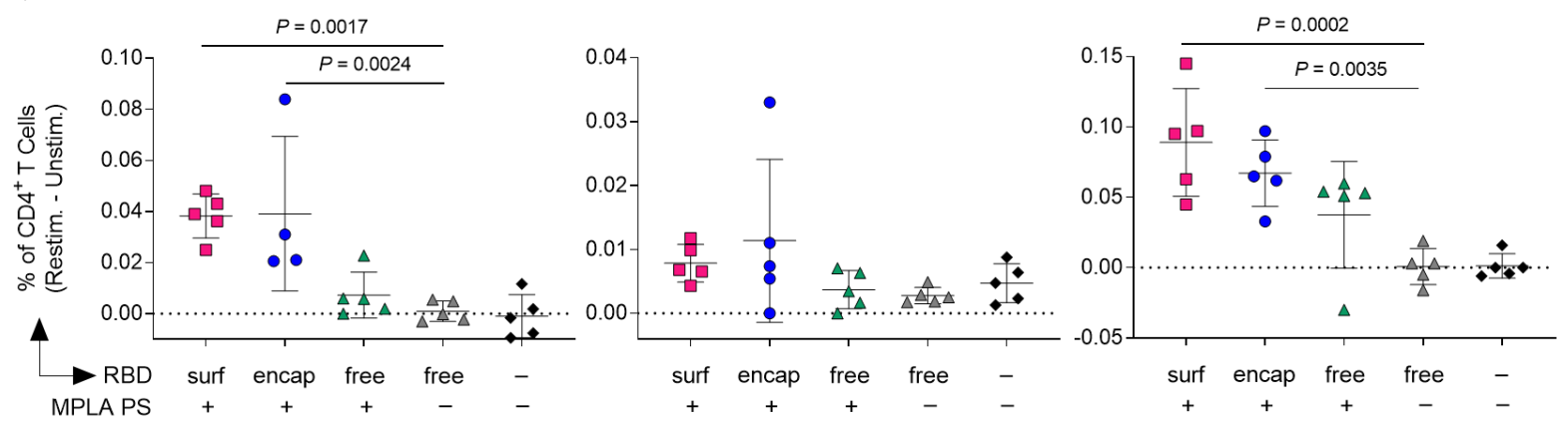

\section{$\underline{3 d}$ ex vivo protein restimulation}

C

IFNy
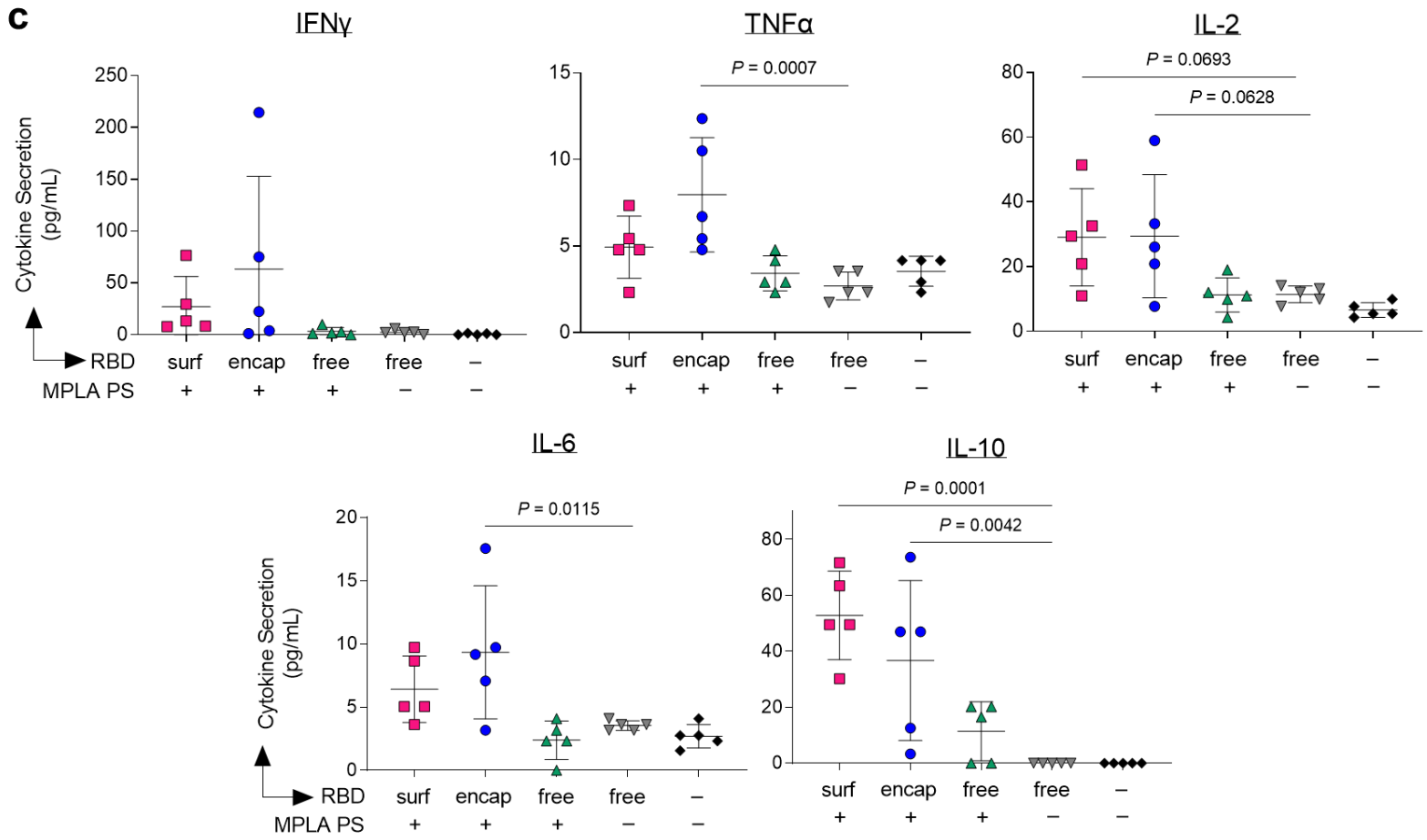
Figure 5 | Vaccination with polymersome-formulated RBD improves antigen-specific T cell responses in mice. Cells isolated from the injection-site draining lymph nodes of PS-vaccinated mice 1 week post-boost were restimulated ex vivo for $6 \mathrm{~h}$ with RBD peptide pools or full RBD protein and analyzed by flow cytometry or multiplexed ELISA, respectively. a-b, percentages of cytokine-positive $\mathrm{CD} 8^{+} \mathrm{T}$ cells (a) and $\mathrm{CD} 4^{+} \mathrm{T}$ cells (b) in response to RBD peptide pools, subtracting unstimulated controls. c, Pro-inflammatory cytokine levels released into the supernatant measured after 3 d restimulation with whole RBD protein. Data plotted as mean \pm SD and represent 1 of 2 experiments with $n=5$ mice each. Symbols represent individual mice. Comparisons to unadjuvanted $\mathrm{RBD}_{\text {free }}$ were made using one-way ANOVA with Dunn's post-test.

\section{DISCUSSION}

In this study, we developed antigen-decorated, oxidation-sensitive polymersomes that mimic virus particles as next-generation nanovaccines. While all adjuvanted formulations generated longlived RBD-binding IgG responses, surface conjugation of antigen was necessary to generate neutralizing antibodies against SARS-CoV-2. More generally, this comparison of antigen formulation on the quality of immune response offers valuable insight into vaccine design, demonstrating the benefits of surface antigen display.

The differences in the immune responses elicited by the two PS antigen formulations, $\mathrm{RBD}_{\text {surf }}$ and $\mathrm{RBD}_{\text {encap }}$, suggest that surface display of antigen leads to stronger GC responses, while PSencapsulated antigen elicits more predominantly an extrafollicular response. Though RBDspecific GC B cells are present in the dLN after treatment with both formulations, a much higher percentage of the RBD-specific $B$ cells recovered after vaccination with $R B D_{\text {surf }}$ exhibited a $G C B$ cell phenotype. This higher percentage could be due to the induction of higher numbers of RBDspecific GC B cells after vaccination with $\mathrm{RBD}_{\text {sur }}$ or an increase in their affinity, leading to easier detection via RBD protein tetramer staining. Both possibilities suggest a more robust GC response, as GC responses are necessary for an efficient SHM leading to increased B cell affinity ${ }^{49}$. Evidence for affinity maturation due to SHM also includes the relatively few epitopes on the RBD linear peptide array to which IgG from $\mathrm{RBD}_{\text {surf }}$-treated groups were specific compared to the other adjuvanted groups. Clonal bursts in GC responses can lead to rapid expansion of highaffinity SHM variants and loss of overall clonal diversity ${ }^{50}$. A difference in the affinity of RBDspecific $\lg G$ generated by these vaccines may also explain the neutralization ability of the plasma after vaccination with $R B D_{\text {surf }}$ but not $R B D_{\text {encap }}$. Further data that suggest that $R B D_{\text {encap }}$ elicits a more extrafollicular response include the fast initial antibody response after priming by $\mathrm{RBD}_{\text {encap }}$, which resulted in RBD-specific IgG AUC 1 week after the initial prime that was significantly higher than that of $\mathrm{RBD}_{\text {surf. In }}$ an extrafollicular $B$ cell response, $B$ cells can differentiate immediately into plasmablasts and begin secreting antibodies after initial $T$ cell help, whereas $B$ cells that enter GCs delay the antibody response by several days ${ }^{45}$. The preferential differentiation into plasmablasts rather than GC B cells was also evident in the increased fraction of plasmablasts within the RBD-specific $B$ cell population generated by $R B D_{\text {encap. }}$ In vaccine development, the generation of robust $\mathrm{GC}$ reactions is preferable to extrafollicular responses because $\mathrm{GC}$ formation will usually result in higher affinity B cells, increased memory B cells and increased long-lived plasma cells in the bone marrow. ${ }^{45}$ 
The difference in mechanism of B cell activation by these two RBD formulations may be explained by previous studies on multimerization of antigen and use of virus-like particles in vaccination. Virus-like particles are multi-protein supra-molecular structures constructed of many identical protein copies. Their multimeric nature has been associated with the induction of potent antibody responses due to $\mathrm{BCR}$ crosslinking in the presence of $\mathrm{CD} 4^{+} \mathrm{T}$ cell help $\mathrm{p}^{51}$. Mechanistic studies by Kato et al. demonstrated that increasing antigen valency can enhance the early activation and proliferation of antigen-specific B cells as well as increase B cell accumulation at the T-B border leading to increased differentiation of antigen-specific B cells into GC B cell and plasma cells ${ }^{52}$. Furthermore, in contrast to $\mathrm{RBD}_{\text {encap }}, \mathrm{RBD}_{\text {surf }}$ most likely efficiently exposes for $\mathrm{BCR}$ interaction the conformational epitopes of RBD reported to be targeted by neutralizing antibodies in plasma of convalescent or vaccinated individuals $\mathrm{s}^{7-10}$. Thus, the multimerization of RBD on the PS surface in addition to its increased availability to B cells led to an improved functional quality of humoral response compared to encapsulated or unformulated RBD.

The type of immune response to SARS-CoV-2 may have important implications in how the infection is cleared and should be considered in vaccine design ${ }^{21}$. Less severe cases of the original SARS-CoV were associated with increased Th1-type cell responses ${ }^{53,54}$. In contrast, Th2type responses were associated with increased pathology due to antibody-dependent enhancement, and several vaccine formulations against SARS-CoV tested in animal models showed signs of immunopathology due to Th2 cell-mediated eosinophil infiltration ${ }^{55,56}$. In this study, we measured the ratio of $\lg \mathrm{G} 1 / \mathrm{lg} \mathrm{g} 2 \mathrm{~b}$ and the cytokine profile of $\mathrm{dLN}$ cells restimulated with RBD protein in order to characterize the type of immune responses generated by our vaccine formulations. $R B D_{\text {encap }}$ and $R B D_{\text {free }}+$ MPLA PS generated lgG2b/lgG1 ratios around 1, suggesting a balance between type 1 and type 2 immunity, whereas RBD surf generated a response with a slightly IgG1-bias, suggestive of a Th2-skewed response. However, both RBD surf and RBD encapf generated significant levels of the Th1-type cytokines IFN $\gamma$, TNF $\alpha$ and IL-2. This Th1-type response is likely induced by the MPLA PS, as MPLA has been shown to generate strong type-1 immunity ${ }^{57}$. Although $\mathrm{RBD}_{\text {surf }}$ generated a slightly lgG1-biased antibody response, it did not result in an increase in Th2-type cytokines upon restimulation compared to $\mathrm{RBD}_{\text {encap. }}$ Combined with the evidence of Th1-type cytokine production upon restimulation, we believe the risk of adverse events related to Th2-type responses is low.

The PS-formulated RBD vaccines were able to generate stronger Th1-driven CD4 ${ }^{+}$and $\mathrm{CD}^{+} \mathrm{T}$ cell responses compared to $\mathrm{RBD}_{\text {free. }}$. We previously demonstrated that as an antigen delivery vehicle, PEG-PPS polymersomes could improve cross-presentation to CD8 ${ }^{+} \mathrm{T}$ cells ${ }^{33}$. This increased T cell response likely occurs because of both enhanced APC targeting as well as rapid endosomal antigen release ${ }^{33}$. APCs are able to accumulate membrane-impermeable nanocarriers such as PS more efficiently than other cell types due to their constitutive macropinocytosis ${ }^{58}$. Once endocytosed, PEG-PPS polymersomes require only a small amount of oxidation to release encapsulated antigen, and payload delivery to the cytosol is not restricted to endosomal compartments with reductive or acidic conditions ${ }^{33}$. Unlike antigen-encapsulated PS, however, acidification may be important for proteolytic degradation of $\mathrm{RBD}_{\text {surf }}$ and translocation to the cytosol, as evidenced by studies on VLPs ${ }^{59,60}$. Additionally, peptides derived from large antigen particles have been found to enter the cross-presentation pathways more efficiently than those derived from soluble antigens, which may provide rationale for the enhanced CD8 ${ }^{+}$

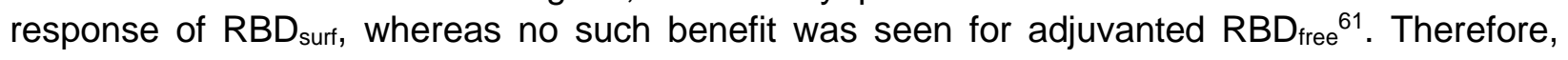
antigen formulation using PS to improve $T$ cell responses could be beneficial in the engineering of future vaccines against cancer or other infectious diseases for which $T$ cell immune responses 
are thought to be necessary for protection, such as herpesviruses, human immunodeficiency virus, and hepatitis $\mathrm{C}$ virus ${ }^{62}$.

In summary, we have demonstrated that a polymersome-based antigen and adjuvant delivery system generates robust humoral immunity and neutralizing antibody titers, as well as $T$ cell responses, against a key SARS-CoV-2 vaccine target, the RBD of Spike. This platform technology is amenable to a wide variety of antigens and formulated or soluble adjuvants. Once the type and dose of adjuvant has been optimized for a given application, a single particle could be used to deliver both antigen and adjuvant to APCs in the injection site-draining lymph node. Additionally, multiple antigens, for example from different viruses or different strains or variants of the same virus, could be conjugated to the same particle as a strategy to induce cross-reactive neutralizing antibodies ${ }^{20}$. Importantly, both surface-decorated and antigen-encapsulated polymersomes remained stable at $4^{\circ} \mathrm{C}$ for at least 6 months, as indicated by consistent particle size and absence of antigen released into solution. Vaccines that exhibit long-term stability without requiring subzero temperatures will likely be important for widespread vaccine distribution, for example to rural populations or developing nations with poor cold chain network. The evaluation of RBD-decorated polymersomes presented here could thus provide insight into the next generation of stable formulations of nanovaccines to combat the current COVID-19 pandemic as well as future viral outbreaks.

\section{METHODS}

\section{RBD production and purification}

For production of the Spike protein RBD (Spike ${ }_{319-541}$; GenBank: MN908947.3), we obtained expression plasmids on pCAGGS backbone containing mammalian codon-optimized sequences for this gene from Florian Krammer's laboratory (Icahn School of Medicine at Mount Sinai, New York, NY) ${ }^{63}$. Suspension-adapted HEK-293F cells were maintained in serum-free Free Style 293 Expression Medium (Gibco). On the day of transfection, cells were inoculated into at a concentration of $1 \times 10^{6}$ cells $\mathrm{mL}^{-1}$. Plasmid DNA (1 mg mL-1) was mixed with linear $25 \mathrm{kDa}$ polyethyleneimine (2 mg mL $\mathrm{mb}^{-1}$; Polysciences) and co-transfected in OptiPRO SFM medium (4\% final concentration; Thermo Fisher). Flasks were cultured in an orbital shaking incubator (135 rpm, $37{ }^{\circ} \mathrm{C}, 5 \% \mathrm{CO}_{2}$ ) for 7 days. Culture medium was then collected by centrifugation, filtered, and loaded into a HisTrap HP $5 \mathrm{~mL}$ column (GE Healthcare) using an ÄKTA pure 25 (GE Healthcare). After washing the column with wash buffer $\left(20 \mathrm{mM} \mathrm{NaH}_{2} \mathrm{PO}_{4}\right.$ and $\left.0.5 \mathrm{M} \mathrm{NaCl}, \mathrm{pH} 8.0\right)$, protein was eluted using a gradient of $500 \mathrm{mM}$ imidazole in wash buffer. The protein was further purified by size-exclusion chromatography using a HiLoad Superdex 200PG column (GE Healthcare) with PBS as an eluent. Dimers of RBD were reduced by the addition of dithiothreitol (1 mM) which was subsequently dialyzed against PBS. All purification steps were carried out at $4{ }^{\circ} \mathrm{C}$. The expressed proteins were verified to be $>90 \%$ pure through SDS-PAGE. The purified proteins were tested for endotoxin using a HEK-Blue ${ }^{\mathrm{TM}}$ TLR4 reporter cell line (InvivoGen), and the endotoxin levels were confirmed to be below $0.01 \mathrm{EU} \mathrm{mL}^{-1}$. Protein concentration was determined by absorbance at $280 \mathrm{~nm}$ using a NanoDrop spectrophotometer (Thermo Scientific). Proteins were stored at a concentration of $4 \mathrm{mg} \mathrm{mL}^{-1}$ at $-80{ }^{\circ} \mathrm{C}$ until use. 


\section{PS formulation}

PEG-PPS polymersomes were formulated by thin film rehydration as previously described ${ }^{33}$. In brief, $20 \mathrm{mg}$ of polymer was dissolved in $750 \mu \mathrm{L}$ dichloromethane (DCM), and DCM was removed by vacuum desiccation overnight. $1 \mathrm{~mL}$ of PBS was then added to the vial, which was rotated at room temperature (RT) for $24 \mathrm{~h}$ to allow complete dispersal of the polymer. The solution was then sequentially extruded through $0.8,0.4,0.2$, and $0.1 \mu \mathrm{m}$ pore membranes (Whatman). To formulate RBD-encapsulated PS, $250 \mu \mathrm{L}$ of PBS containing $4 \mathrm{mg} / \mathrm{mL}$ RBD was added to the polymer thin film for rehydration, and the solution was rotated at $4{ }^{\circ} \mathrm{C}$ for $72 \mathrm{~h}$ before extrusion as above. After extrusion, RBD-encapsulated polymersomes were passed through a sepharaose size exclusion chromatography (SEC) column to remove unencapsulated free RBD. The RBD content was quantified by SDS-PAGE using mini-protein TGX stain-free precast gels (Bio-Rad). Gels were imaged on a ChemiDoc XRS+ Gel Documentation System (Bio-Rad) and analyzed using ImageJ.

RBD-surface-conjugated PS were synthesized by first formulating empty PS as above consisting of $25 \% \mathrm{~N}_{3}$-PEG-PPS. RBD was conjugated to a sulfo DBCO-Maleimide linker (Click Chemistry Tools) at the molar ratio of 1:5 (RBD:linker) and reacted for 2.5 hours. Unconjugated linker was removed by Zeba spin desalting columns (7K MWCO; ThermoFisher). The resulting RBD-linker was analyzed by MALDI-TOF MS using an a-cyano-4-hydroxycinnamic acid (Sigma-Aldrich) matrix and a Bruker ultrafleXtreme MALDI TOF/TOF instrument. RBD-linker (4 wt\% of polymersome) was then incubated with empty $\mathrm{N}_{3}$-PEG-PPS polymersomes overnight at $\mathrm{RT}$. RBD-surface-conjugated PS were then passed through SEC to remove unconjugated RBD-linker. The conjugation was monitored by SDS-PAGE, and the final RBD content was quantified by the CBQCA Protein Quantitation Kit (ThermoFisher). Fluorescently labeled PS were prepared by conjugating AlexaFluor-647 alkyne dye (ThermoFisher) to PS containing $25 \% \mathrm{~N}_{3}$-PEG-PPS. The dye was mixed with PS at a molar ratio of 1:20 (dye:N $\mathrm{N}_{3}-\mathrm{PEG}-\mathrm{PPS}$ ), and the solution was stirred overnight at RT. Labeled PS were then passed through SEC to remove unconjugated dye. To

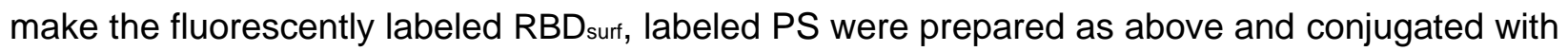
RBD as described, followed by SEC purification. Final RBD content was quantified by the CBQCA Protein Quantitation Kit (ThermoFisher).

MPLA PS were fabricated by flash nanoprecipitation using a 3D printed impingement jets mixer ${ }^{64}$. $20 \mathrm{mg}$ PEG-PPS and $2 \mathrm{mg}$ MPLA (PHADß; Avanti Polar Lipids) were dissolved in $500 \mu \mathrm{L}$ tetrahydrofuran (THF) and loaded into a $1 \mathrm{~mL}$ plastic disposable syringe. $500 \mu \mathrm{L}$ PBS was loaded into a second syringe, and the two solutions were impinged against one another slowly within the mixer by hand. The impinged solution was immediately vortexed to form a homogenous polymersome solution which was then extruded and purified by SEC as described above. MPLA loading was quantified using a liquid chromatography-tandem mass spectrometry (LC-MS/MS) method as previously described ${ }^{65}$ using PHAD®-504 (Avanti Polar Lipids) as an internal standard on an Agilent 6460 Triple Quad MS-MS with 1290 UHPLC. 


\section{PS characterization}

The size and polydispersity index (PDI) of all the polymersome formulations were measured by dynamic light scattering (DLS) using a Zetasizer Nano ZS90 (Malvern Instruments). Cryogenic electron microscopy (cryoEM) images were obtained on a FEI Talos 200kV cryoEM dedicated electron microscope.

\section{MPLA PS in vitro activity}

To determine TLR4 activation, HEK-Blue ${ }^{T M}$ TLR4 cells (Invivogen) were incubated with increasing concentrations of MPLA PS for $24 \mathrm{~h} 37{ }^{\circ} \mathrm{C}$ in a $5 \% \mathrm{CO}_{2}$ incubator. NF-kB-induced SEAP activity was detected using QUANTI-Blue ${ }^{\mathrm{TM}}$ (Invivogen) and by reading the OD at $650 \mathrm{~nm}$. For dendritic cell activation experiments, BMDCs were prepared from C57BI/6 mice (Jackson) as previously described. ${ }^{66}$ After 9 days of culture, cells were seeded at $2 \times 10^{5}$ cells/well in round-bottom 96well plates (Fisher Scientific) in IMDM with 10\% FBS and $2 \%$ penicillin/streptomycin (Life Technologies). Cells were treated with varying concentrations of MPLA PS or free MPLA and incubated for $24 \mathrm{~h}$ at $37^{\circ} \mathrm{C}$ in a $5 \% \mathrm{CO}_{2}$ incubator. After $24 \mathrm{~h}$, the supernatant was collected, and cytokine concentration was measured using a multiplexed mouse Th cytokine panel (BioLegend) according to the manufacturer's instructions.

\section{$\mathrm{RBD}_{\text {surf }}$ in vitro activity}

For the cell-based hACE2-binding assay, human embryonic kidney (HEK)-293T cells overexpressing human ACE-2 (HEK-hACE2) were obtained from BEI Resources (NIH NIAID). Fluorescently labeled empty $\mathrm{PS}, \mathrm{RBD}_{\text {surf, }}$ or $\mathrm{RBD}_{\text {free }}$ was incubated at varying concentrations with $5 \times 10^{4} \mathrm{HEK}$-hACE2 cells at $4^{\circ} \mathrm{C}$ for $20 \mathrm{~min}$. Cells were then washed three times in PBS with $2 \%$ FBS. Binding of RBD to hACE-2 on the cell surface was assessed via the mean fluorescent intensity measured by flow cytometry using BD LSRFortessa (BD Biosciences).

\section{Production of RBD protein tetramers}

RBD protein expressed with AviTag was purchased from GenScript. Site-specific biotinylation of the AviTag was performed using BirA Biotin-Protein Ligase Reaction kit (Avidity). Next, unconjugated biotin was removed using Zeba spin desalting columns, 7K MWCO (ThermoFisher). The quantification of reacted biotin was performed using the Pierce Biotin Quantification Kit (ThermoFisher). Biotinylated RBD was incubated with either streptavidin-conjugated PE (Biolegend) or streptavidin-conjugated APC fluorophores (Biolegend) for 20 min on ice at a molar ratio of 4:1 of biotin to streptavidin. Streptavidin-conjugated FITC (BioLegend) was reacted with excess free biotin to form a non-RBD-specific streptavidin probe as a control. Tetramer formation was confirmed using SDS-PAGE gel. Cells were stained for flow cytometry with all three streptavidin probes at the same time as other fluorescent surface markers at a volumetric ratio of 1:100 for RBD-streptavidin-PE and 1:200 for RBD-streptavidin-APC and biotin-streptavidin-FITC. 


\section{Mouse vaccination experiments}

All experiments were performed in accordance with the Institutional Animal Care and Use Committee at the University of Chicago. Female 8-week-old C57BL/6 mice (Jackson Laboratory) were randomly assigned to cohorts of $n=5$ and vaccinated with $10 \mu \mathrm{g}$ of antigen and $5 \mu \mathrm{g}$ of adjuvant s.c. in the hock (either 2 or 4 hocks) and boosted on day 21. On day 7, 14, 20, and 28 post vaccination, $100 \mu \mathrm{L}$ of blood was collected in EDTA-K2-coated tubes (Milian), and plasma was separated by centrifugation and stored at $-80^{\circ} \mathrm{C}$ until use. On day 28 after initial vaccination, mice were sacrificed. Splenocytes and lymph node cells from draining lymph nodes were collected. Single-cell suspensions of the lymph node were prepared by digestion in collagenase $\mathrm{D}$ for $45 \mathrm{~min}$ at $37^{\circ} \mathrm{C}$. Splenocytes and lymph node cells were filtered through a $70 \mu \mathrm{m}$ cell strainer. Splenocytes were then incubated in ACK lysis buffer to remove red blood cells. Lymph node cells were stained for $T_{\text {fh }}$ cells and RBD-specific $B$ cells using fluorescent probes listed in Supplementary Tables 1 and 2, respectively. Samples were acquired on a BD LSR-Fortessa (BD) and analyzed using FlowJo ${ }^{\mathrm{TM}}$ software. Representative gating strategies used to identify the cell populations are shown in Supplemental Figures 14 ( $T_{\text {fh }}$ cells) and 15 (RBD-specific B cells).

To assess antigen-specific cytokine production by T cells, $1 \times 10^{6}$ lymph node cells were incubated with pools of 15-mer peptides overlapping by 10 amino acids covering the $\mathrm{N}$-terminus of SARS-CoV-2 Spike protein up to the furin cleavage site (S1 pool; PepMix SARS-CoV-2 Spike Glycoprotein, JPT) for $6 \mathrm{~h}$ at $37^{\circ} \mathrm{C}$ with $5 \% \mathrm{CO}_{2}$. Monensin (GolgiStop, BD) was added after $2 \mathrm{~h}$ of incubation to inhibit cytokine secretion. Cells were stained for surface markers using fluorescent monoclonal antibodies (mAbs). Cells were subsequently fixed and permeabilized using BD Cytofix/Cytoperm ${ }^{\mathrm{TM}}$, and intracellular cytokines were stained using fluorescent-mAbs listed in Supplementary Table 3. Samples were acquired on a BD LSR-Fortessa (BD) and analyzed using FlowJo ${ }^{T M}$ software. A representative gating strategy used to identify the cell populations is shown in Supplementary Figure 19. To assess antigen-specific cytokine secretion, lymph node cells were plated at $5 \times 10^{5}$ cells/well were incubated with $100 \mu \mathrm{g} \mathrm{RBD}$ for $3 \mathrm{~d}$ at $37^{\circ} \mathrm{C}$ with $5 \% \mathrm{CO}_{2}$. After 3 $\mathrm{d}$, the supernatant was analyzed for cytokine concentration via a multiplexed mouse Th cytokine panel (BioLegend) according to the manufacturer's instructions. Samples were aquired on an Attune NxT flow cytometer (ThermoFisher), and analyzed with LEGENDplex v8.0 software.

For long-term experiments, mice were not sacrificed but continued to be bled on day 2, 7, 14 and then every 2 weeks for 80 days.

\section{RBD-binding ELISA}

Plasma was assessed for anti-RBD IgG and IgA by ELISA. 96-well ELISA plates (Costar highbind flat-bottom plates, Corning) were coated with $10 \mu \mathrm{g} / \mathrm{mL}$ RBD in carbonate buffer (50 mM sodium carbonate/sodium bicarbonate, $\mathrm{pH} 9.6$ ) overnight at $4{ }^{\circ} \mathrm{C}$. The following day, plates were washed three times in PBS with $0.05 \%$ Tween 20 (PBS-T) and then blocked with $1 \mathrm{x}$ casein (Sigma) for $1 \mathrm{~h}$ at RT. Following blocking, wells were washed three times with PBS-T and further incubated with six 10 -fold dilutions of plasma in $1 \mathrm{x}$ casein for $2 \mathrm{~h}$ at $\mathrm{RT}$. Wells were then washed five times with PBS-T and incubated for an additional hour at RT with horseradish peroxide (HRP)conjugated antibodies against mouse IgG, IgG1, IgG2b, IgG3, or IgA (Southern Biotech). After 
five washes with PBS-T, bound RBD-specific Ig was detected with tetramethylbenzidine (TMB) substrate. Stop solution $\left(3 \% \mathrm{H}_{2} \mathrm{SO}_{4}+1 \% \mathrm{HCl}\right)$ was added after 18 min of TMB incubation at RT, and the OD was measured at 450 and $570 \mathrm{~nm}$ on an Epoch Microplate Spectrophotometer (BioTek). Background signal at $570 \mathrm{~nm}$ was subtracted from the OD at $450 \mathrm{~nm}$. Fold-change over the average of blank wells was then calculated and log-transformed. The area under the curve (AUC) of log-transformed fold change versus log-transformed dilution was then calculated.

\section{RBD-binding IgG ELISpot assay}

ELISpot plates (Millipore IP Filter plate) were coated with $20 \mu \mathrm{g} / \mathrm{mL}$ RBD in sterile PBS overnight at $4{ }^{\circ} \mathrm{C}$. Plates were then blocked using ELISpot Media (RPMI 1640, $1 \%$ glutamine, 10\% fetal bovine serum, $1 \%$ penicillin-streptomycin) for 2 hours at $37^{\circ} \mathrm{C}$. Splenocytes from vaccinated mice were seeded in triplicate at a starting concentration of $6.75 \times 10^{5}$ cell/well and diluted seven times in 3-fold serial dilutions. Plates were incubated for 18 hours at $37^{\circ} \mathrm{C}$ and $5 \% \mathrm{CO}_{2}$ after which the cells were washed five times in PBS. Wells were incubated with $100 \mu \mathrm{L}$ lgG-biotin HU adsorbed (Southern Biotech) for $2 \mathrm{~h}$ at RT. Next, plates were washed four times in PBS followed by the addition of $100 \mu \mathrm{L}$ HRP-conjugated streptavidin/well for $1 \mathrm{~h}$ at RT. Plates were washed again and incubated with $100 \mu \mathrm{L} \mathrm{TMB} /$ well for 5 minutes until distinct spots emerged. Finally, plates are then washed three times with distilled water and left to dry completely in a laminar flow hood. A CTL ImmunoSpot Analyzer was used to image plates, count spots, and perform quality control.

\section{SARS-CoV-2 virus neutralization assay}

SARS-CoV-2 viruses (400 plaque forming units; strain nCov/Washington/1/2020, provided by the National Biocontainment Laboratory, Galveston TX, USA) were incubated with 4-fold serial dilutions of heat-inactivated plasma from vaccinated or control mice and for $1 \mathrm{~h}$ at $37^{\circ} \mathrm{C}$ in DMEM with $2 \%$ fetal bovine serum, penicillin-streptomycin (100 U/mL penicillin, $100 \mu \mathrm{g} / \mathrm{mL}$ streptomycin), and non-essential amino acids (10 mM, glycine, L-alanine, L-asparagine, L-aspartic acid, Lglutamic acid, L-proline, L-serine; Gibco). The pre-incubated viruses were then applied to VeroE6 cell monolayers, and the cells were maintained until $>90 \%$ cell death for the negative control (4-5 d). Cells were then washed with PBS, fixed with $10 \%$ formalin, stained with crystal violet, and quantified with a Tecan infinite m200 microplate reader (excitation/emission $592 \mathrm{~nm} / 636 \mathrm{~nm}$ ). Neutralization titer is measured as the greatest dilution that inhibits $50 \%$ of SARS-CoV- 2 induced cell death (EC50). To determine the EC50, data were fit using a least squares variable slope fourparameter model. To ensure realistic EC50 values, we considered a dilution (1/X) of $X=10^{-1}$ to be $100 \%$ neutralizing and a dilution of $X=10^{8}$ to be $0 \%$ neutralizing and constrained EC50 $>0$. Plasma from convalescent human COVID-19 patients were provided by Ali Ellebedy (Washington University School of Medicine, St. Louis, MO; Catalog \# NR-53661, NR-53662, NR-53663, NR53664, and NR-53665). 


\section{Peptide array analysis}

Antibody specificity to linear epitopes of the spike protein was analyzed using a CelluSpots ${ }^{\mathrm{TM}}$ Covid19_hullB Peptide Array (Intavis Peptide Services, Tubingen, Germany) according to the manufacturer's protocol. The array comprises 254 peptides spanning the full-length sequence of the Spike protein (NCBI GenBank accession \# QHD43416.1), with each 15-mer peptide offset from the previous one by 5 amino acids. Briefly, peptide arrays were blocked in $1 x$ casein solution at $4{ }^{\circ} \mathrm{C}$ overnight. Arrays were then incubated with pooled serum diluted 1:200 in $1 \times$ casein for 6 $\mathrm{h}$ at RT on an orbital shaker (60 rpm). After $6 \mathrm{~h}$, arrays were washed four times with PBS-T and incubated for an addition $2 \mathrm{~h}$ at RT, $60 \mathrm{rpm}$ with goat anti-mouse IgG conjugated to HRP (Southern Biotech) diluted 1:5000 in 1x casein. Arrays were washed another four times with PBST. Spots were detected with Clarity ${ }^{\mathrm{TM}}$ Western ECL Substrate (Bio-Rad), and chemiluminescence was measured using a ChemiDoc XRS+ Gel Documentation System (Bio-Rad). Spots were analyzed using Spotfinder software (version v3.2.1).

\section{Software packages and statistical analysis}

Statistical analysis was performed using GraphPad Prism 9 (GraphPad). Data were analyzed using one-way ANOVA with Dunn's or Tukey's post-hoc correction for multiple hypothesis testing unless otherwise stated. All flow cytometry data were analyzed using FlowJo_v10.7.2 software (FlowJo LLC, BD Biosciences). Figures 1 and 2 were created using BioRender (https://biorender.com) as part of an Academic License through the Chicago Immunoengineering Innovation Center.

\section{ACKNOWLEDGMENTS}

We are grateful for funding support through a pilot project grant from the Chicago Immunoengineering Innovation Center of the University of Chicago, as well as the Chicago Biomedical Consortium COVID-19 Response Award (\# CR-002) to M.A.S. In addition, a number of researchers were supported by fellowships via the NIH NHLBI (\# T32-HL007605 to L.R.V.), Canadian Institutes of Health Research (\#201910MFE-430736-73744 to N.M.), University of Chicago Comprehensive Cancer Center (Sigal Fellowship in Immuno-Oncology to N.M.), NIH NCI (\# F30-CA221250 to M.R.S.), NIH NIGMS (\# T32-GM007281 to M.R.S.) and NIH NIAID (\# T32AI007090 to T.M.M. and A.C.T.). We are grateful to the laboratory of Florian Krammer (Icahn School of Medicine at Mount Sinai, New York City, NY) for providing plasmids coding for the Spike RBD, produced with support from the NIH NIAID (Contract \# HHSN272201400008C). We are also grateful to the groups of Jesse Bloom (Fred Hutchinson Cancer Research Center, Seattle, WA) and Ali Ellebedy (Washington University School of Medicine, St. Louis, MO) for contributing reagents via the NIH NIAID BEI Resources repository. We acknowledge helpful discussions with Patrick C. Wilson, Jenna J. Guthmiller, Anne I. Sperling, and Aaron Esser-Kahn (University of Chicago, Chicago, IL) and with Robert Baker and David Boltz (Illinois Institute of Technology Research Institute, Chicago, IL) that were instrumental to experimental planning and model development. We acknowledge Suzana Gomes and Tera Lavoie for technical assistance. Parts of this work were carried out at the Cytometry and Antibody Technology Core Facility (Cancer Center Support Grant P30CA014599), the Soft Matter Characterization Facility, the Mass 
Spectrometry Facility (NSF instrumentation grant CHE-1048528), the Nuclear Magnetic Resonance Facility, the Advanced Electron Microscopy Facility (RRID:SCR_019198), and the Human Immunologic Monitoring Facility (RRID:SCR_017916) at the University of Chicago.

\section{COMPETING INTERESTS}

M.A.S. and J.A.H. have patents related to the polymersome technology and interests in LantaBio, which has licensed those patents.

\section{REFERENCES}

1. Le, T. et al. Evolution of the COVID-19 vaccine development landscape. Nature Review Drug Discovery 19, 667-668 (2020).

2. Kyriakidis, N. C. et al. SARS-CoV-2 vaccines strategies: a comprehensive review of phase 3 candidates. NPJ Vaccines 6, 28 (2021).

3. Richmond, P. et al. Safety and immunogenicity of S-Trimer (SCB-2019), a protein subunit vaccine candidate for COVID-19 in healthy adults: a phase 1, randomised, double-blind, placebocontrolled trial. The Lancet 397, 682-694 (2021).

4. Keech, C. et al. Phase 1-2 Trial of a SARS-CoV-2 Recombinant Spike Protein Nanoparticle Vaccine. New England Journal of Medicine 383, 2320-2332 (2020).

5. Zhou, P. et al. A pneumonia outbreak associated with a new coronavirus of probable bat origin. Nature 579, 270-273 (2012).

6. Lan, J. et al. Structure of the SARS-CoV-2 spike receptor-binding domain bound to the ACE2 receptor. Nature 581, 215-220 (2020).

7. Cao, Y. et al. Potent Neutralizing Antibodies against SARS-CoV-2 Identified by High-Throughput Single-Cell Sequencing of Convalescent Patients' B Cells. Cell 182, 73-84.e16 (2020).

8. Zost, S. J. et al. Potently neutralizing and protective human antibodies against SARS-CoV-2. Nature 584, 443-449 (2020).

9. Shi, R. et al. A human neutralizing antibody targets the receptor-binding site of SARS-CoV-2. Nature 584, 120-124 (2020).

10. Liu, L. et al. Potent neutralizing antibodies against multiple epitopes on SARS-CoV-2 spike. Nature 584, 450-456 (2020).

11. Sternberg, A \& Naujokat, C.. Structural features of coronavirus SARS-CoV-2 spike protein: Targets for vaccination. Life Sciences 257, 118056 (2020).

12. Dai, L. \& Gao, G. F. Viral targets for vaccines against COVID-19. Nature Reviews Immunology 21, 73-82 (2021).

13. Wang, Y., Wang, L., Cao, H. \& Liu, C. SARS-CoV-2 S1 is superior to the RBD as a COVID-19 subunit vaccine antigen. Journal of Medical Virology 93, 892-898 (2021).

14. Irvine, D. J. \& Read, B. J. Shaping humoral immunity to vaccines through antigen-displaying nanoparticles. Current Opinion in Immunology 65 1-6 (2020).

15. Shin, M. D. et al. COVID-19 vaccine development and a potential nanomaterial path forward. Nature Nanotechnology 15 646-655 (2020).

16. Singh, A. Eliciting B cell immunity against infectious diseases using nanovaccines. Nature Nanotechnology 16, 16-24 (2021).

17. Kit Tan, T. et al. A COVID-19 vaccine candidate using SpyCatcher multimerization of the SARSCoV-2 spike protein receptor-binding domain induces potent neutralising antibody responses. Nature Communications 12, 1-16 (2021).

18. Kang, Y.-F. et al. Rapid Development of SARS-CoV-2 Spike Protein Receptor-Binding Domain Self-Assembled Nanoparticle Vaccine Candidates. ACS Nano 15, 2738-2752 (2021). 
19. Walls, A. C. et al. Elicitation of Potent Neutralizing Antibody Responses by Designed Protein Nanoparticle Vaccines for SARS-CoV-2. Cell 183, 1367-1382.e17 (2020).

20. Cohen, A. A. et al. Mosaic nanoparticles elicit cross-reactive immune responses to zoonotic coronaviruses in mice. Science 371, 735-741 (2021).

21. Jeyanathan, M. et al. Immunological considerations for COVID-19 vaccine strategies. Nature Reviews Immunology 20, 615-632 (2020).

22. Cox, R. J. \& Brokstad, K. A. Not just antibodies: B cells and T cells mediate immunity to COVID19. Nature Reviews Immunology 20, 581-582 (2020).

23. Sette, A. \& Crotty, S. Adaptive immunity to SARS-CoV-2 and COVID-19. Cell 184 861-880 (2021).

24. Chen, Z. \& John Wherry, E. T cell responses in patients with COVID-19. Nature Reviews Immunology. 20 529-536 (2020).

25. Sekine, T. et al. Robust T Cell Immunity in Convalescent Individuals with Asymptomatic or Mild COVID-19. Cell 183, 158-168.e14 (2020).

26. Grifoni, A. et al. Targets of T Cell Responses to SARS-CoV-2 Coronavirus in Humans with COVID-19 Disease and Unexposed Individuals. Cell 181, 1489-1501.e15 (2020).

27. Peng, Y. et al. Broad and strong memory CD4+ and CD8+ T cells induced by SARS-CoV-2 in UK convalescent individuals following COVID-19. Nature Immunology 21, 1336-1345 (2020).

28. Bonifacius, A. et al. COVID-19 immune signatures reveal stable antiviral T cell function despite declining humoral responses. Immunity 54, 340-354.e6 (2021).

29. Caserta, S. et al. Reduction and Functional Exhaustion of T Cells in Patients With Coronavirus Disease 2019 (COVID-19). Front. Immunol 11, 827 (2019).

30. Chen, G. et al. Clinical and immunological features of severe and moderate coronavirus disease 2019. Journal of Clinical Investigation 130, 2620-2629 (2020).

31. Altmann, D. M. \& Boyton, R. J. SARS-CoV-2 T cell immunity: Specificity, function, durability, and role in protection. Science Immunology. 56160 (2020).

32. Napoli, A., et al. Oxidation-responsive polymeric vesicles. Nature Materials 3, 183-189 (2004).

33. Scott, E. A. et al. Dendritic cell activation and T cell priming with adjuvant- and antigen-loaded oxidation-sensitive polymersomes. Biomaterials 33, 6211-6219 (2012).

34. Cerritelli, S. et al. Aggregation Behavior of Poly(ethylene glycol-bl-propylene sulfide) Di-and Triblock Copolymers in Aqueous Solution. Langmuir 25, 11328-11335 (2009).

35. Stano, A., et al. Tunable T cell immunity towards a protein antigen using polymersomes vs. solidcore nanoparticles. Biomaterials 34, 4339-4346 (2013).

36. Galan-Navarro, C. et al. Oxidation-sensitive polymersomes as vaccine nanocarriers enhance humoral responses against Lassa virus envelope glycoprotein. Virology 512, 161-171 (2017).

37. Zhu, N. et al. A Novel Coronavirus from Patients with Pneumonia in China, 2019. New England Journal of Medicine 382, 727-733 (2020).

38. Stevens, T. L. et al. Regulation of antibody isotype secretion by subsets of antigen-specific helper T cells. Nature 334, 255-258 (1988).

39. Chao, Y. X., Rötzschke, O. \& Tan, E.-K. The role of IgA in COVID-19. Brain, Behavior, and Immunity 87, 182-183 (2020).

40. Renegar, K. B., et al. Role of IgA versus IgG in the control of influenza viral infection in the murine respiratory tract. The Journal of Immunology 173, 1978-1986 (2004).

41. Hinton, D. M. Convalescent Plasma EUA Letter of Authorization. (March 9, 2021).

42. Yi, C. et al. Key residues of the receptor binding motif in the spike protein of SARS-CoV-2 that interact with ACE2 and neutralizing antibodies. Cellular and Molecular Immunology 17, 621-630 (2020).

43. Shang, J. et al. Structural basis of receptor recognition by SARS-CoV-2. Nature 581, 221-224 (2020).

44. Choi, Y. S. et al. ICOS Receptor Instructs T Follicular Helper Cell versus Effector Cell Differentiation via Induction of the Transcriptional Repressor Bcl6. Immunity 34, 932-946 (2011). 
45. Akkaya, M., Kwak, K. \& Pierce, S. K. B cell memory: building two walls of protection against pathogens. Nature Reviews Immunology 20, 229-238 (2020).

46. Toyama, H. et al. Memory B Cells without Somatic HypermutationAre Generated from Bcl6Deficient B Cells. Immunity 17, 329-339 (2002).

47. Mocellin, S., Marincola, F. M. \& Young, H. A. Interleukin-10 and the immune response against cancer: a counterpoint. Journal of Leukocyte Biology 78, 1043-1051 (2005).

48. Kishimoto, T. Interleukin-6: discovery of a pleiotropic cytokine. Arthritis Research \& Therapy 8, (2006).

49. Cyster, J. G. \& Allen, C. D. C. B Cell Responses: Cell Interaction Dynamics and Decisions. Cell 177 524-540 (2019).

50. Mesin, L., Ersching, J. \& Victora, G. D. Germinal Center B Cell Dynamics. Immunity 45 471-482 (2016).

51. Mohsen, M. O. et al. Major findings and recent advances in virus-like particle (VLP)-based vaccines. Seminars in Immunology 34, 123-132 (2017).

52. Kato, Y. et al. Multifaceted Effects of Antigen Valency on B Cell Response Composition and Differentiation In Vivo. Immunity 53, 548-563.e8 (2020).

53. Oh, H. et al. Understanding the T cell immune response in SARS coronavirus infection. Emerging Microbes and Infections 1, 1-6 (2012).

54. Shin, H.-S. et al. Immune Responses to Middle East Respiratory Syndrome Coronavirus During the Acute and Convalescent Phases of Human Infection. Clinical Infectious Diseases 68, 984-992 (2019).

55. Yasui, F. et al. Prior Immunization with Severe Acute Respiratory Syndrome (SARS)-Associated Coronavirus (SARS-CoV) Nucleocapsid Protein Causes Severe Pneumonia in Mice Infected with SARS-CoV. The Journal of Immunology 181, 6337-6348 (2008).

56. Liu, L. et al. Anti-spike lgG causes severe acute lung injury by skewing macrophage responses during acute SARS-CoV infection. JCI Insight 4, (2019).

57. Casella, C. R. \& Mitchell, T. C. Putting endotoxin to work for us: Monophosphoryl lipid A as a safe and effective vaccine adjuvant. Cellular and Molecular Life Sciences 65, 3231-3240 (2008).

58. Sallusto, F. et al. Dendritic cells use macropinocytosis and the mannose receptor to concentrate macromolecules in the major histocompatibility complex class II compartment: downregulation by cytokines and bacterial products. Journal of Experimental Medicine 182, 389-400 (1995).

59. Leclerc Víctor, C. et al. Endosome-to-Cytosol Pathway Virus-Like Particles Using an In Vivo, Dendritic Cells Can Cross-Present. Journal of Immunology References 171, 2242-2250 (2003).

60. Bachmann, M. F. \& Jennings, G. T. Vaccine delivery: a matter of size, geometry, kinetics and molecular patterns. Nature Reviews Immunology 10, 787-796 (2010).

61. Gamvrellis, A. et al. Vaccines that facilitate antigen entry into dendritic cells. Immunology and Cell Biology 82 506-516 (2004).

62. Quiroga, M. F. et al. Features of effective T Cell-inducing vaccines against chronic viral infections. Frontiers in Immunology 9, 276 (2018).

63. Amanat, F. et al. A serological assay to detect SARS-CoV-2 seroconversion in humans. Nature Medicine 26, 1033-1036 (2020).

64. Allen, S., Osorio, O., Liu, Y. G. \& Scott, E. Facile assembly and loading of theranostic polymersomes via multi-impingement flash nanoprecipitation. Journal of Controlled Release 262, 91-103 (2017).

65. Hamdy, S., Haddadi, A., Somayaji, V., Ruan, D. \& Samuel, J. Pharmaceutical analysis of synthetic lipid A-based vaccine adjuvants in poly (d,I-lactic-co-glycolic acid) nanoparticle formulations. Journal of Pharmaceutical and Biomedical Analysis 44, 914-923 (2007).

66. Lutz, M. B. et al. An advanced culture method for generating large quantities of highly pure dendritic cells from mouse bone marrow. Journal of Immunological Methods 223, 77-92 (1999). 


\section{SUPPLEMENTARY INFORMATION}

\section{Polymersomes decorated with SARS-CoV-2 spike protein receptor binding domain elicit robust humoral and cellular immunity}

Lisa R. Volpatti ${ }^{*}$, Rachel P. Wallace ${ }^{1^{*}}$, Shijie Cao ${ }^{1^{*}}$, Michal M. Raczy ${ }^{1^{*}}$, Ruyi Wang ${ }^{1^{*}}$, Laura T. Gray $^{1}$, Aaron T. Alpar ${ }^{1}$, Priscilla S. Briquez ${ }^{1}$, Nikolaos Mitrousis ${ }^{1}$, Tiffany M. Marchell ${ }^{2}$, Maria Stella Sasso ${ }^{1}$, Mindy Nguyen ${ }^{1}$, Aslan Mansurov ${ }^{1}$, Erica Budina ${ }^{1}$, Ani Solanki ${ }^{3}$, Elyse A. Watkins ${ }^{1}$, Mathew R. Schnorenberg ${ }^{1}$, Andrew C. Tremain ${ }^{2}$, Joseph W. Reda ${ }^{1}$, Vlad Nicolaescu ${ }^{4}$, Kevin Furlong ${ }^{4}$, Steve Dvorkin ${ }^{4}$, Shann S. Yu ${ }^{1}$, Balaji Manicassamy ${ }^{5}$, James L. LaBelle ${ }^{6}$, Matthew V. Tirrell ${ }^{1,7}$, Glenn Randall, ${ }^{4}$ Marcin Kwissa ${ }^{1}$, Melody A. Swartz ${ }^{1,2,8,9+}$, Jeffrey A. Hubbell ${ }^{1,2,9+}$

*These authors contributed equally to this work

${ }^{1}$ Pritzker School of Molecular Engineering, University of Chicago, Chicago, IL 60637, United States

${ }^{2}$ Committee on Immunology, University of Chicago, Chicago, IL 60637, United States

${ }^{3}$ Animal Resources Center, University of Chicago, Chicago, IL 60637, United States

${ }^{4}$ Department of Microbiology, Howard T. Ricketts Laboratory, University of Chicago, Chicago, IL 60637, United States

${ }^{5}$ Department of Microbiology and Immunology, University of lowa, lowa City, IA 52242, United States

${ }^{6}$ Department of Pediatrics, University of Chicago Comer Children's Hospital, Chicago, IL 60637, United States

${ }^{7}$ Materials Science Division, Argonne National Laboratory, Lemont, IL 60439, United States

${ }^{8}$ Ben May Department of Cancer Research, University of Chicago, Chicago, IL 60637, United States

${ }^{9}$ Committee on Cancer Biology, University of Chicago, Chicago, IL 60637, United States

+Correspondence to melodyswartz@uchicago.edu, jhubbell@uchicago.edu 


\section{Table of Contents}

Supplementary Figure $\mathbf{S} 1$. Synthesis and characterization of $\mathrm{N}_{3}$-PEG-PPS.

Supplementary Figure S2. Synthesis and characterization of RBD-linker.

Supplementary Figure S3. Synthesis and characterization of PEG-PPS.

Supplementary Figure S4. Additional cryoEM images of PS.

Supplementary Figure S5. PS stability by SDS PAGE after $>180 \mathrm{~d}$ at $4{ }^{\circ} \mathrm{C}$.

Supplementary Figure S6. RBD binding to HEK-hACE2 and HEK-293 cells.

Supplementary Figure S7. MPLA PS as a TLR4 agonist.

Supplementary Figure S8. In vitro activity of MPLA PS.

Supplementary Figure S9. ELISA absorbance vs. dilution curves.

Supplementary Figure S10. Presence of IgA antibodies.

Supplementary Figure S11. IgG antibody and viral neutralization titers.

Supplementary Figure S12. Representative peptide array images.

Supplementary Figure $\mathbf{S 1 3}$. Analysis of plasma by mice vaccinated with $\mathrm{RBD}_{\text {sur }}+\mathrm{RBD}_{\text {encap }}+$ MPLA PS and RBD free $+M P L A_{\text {free }}$.

Supplementary Figure S14. Representative T follicular helper cell gating strategy.

Supplementary Figure S15. Representative B cell gating strategy.

Supplementary Figure S16. Total and naïve B cells in vaccinated mice 1 week post-boost.

Supplementary Figure S17. Representative RBD protein tetramer staining.

Supplementary Figure S18. RBD-specific cells in vaccinated mice 1 week post-boost.

Supplementary Figure S19. Representative intracellular cytokine gating strategy.

Supplementary Figure S20. Th2-type cytokines secreted upon ex vivo stimulation with RBD.

Supplementary Table S1. Summary of loading capacities of PS.

Supplementary Table S2. Probes and antibodies for $\mathrm{T}_{\text {fh }}$ cell panel.

Supplementary Table S3. Probes and antibodies for RBD-specific B cell panel.

Supplementary Table S4. Probes and antibodies for restimulation panel.

Chemical Synthesis and Characterization 
a

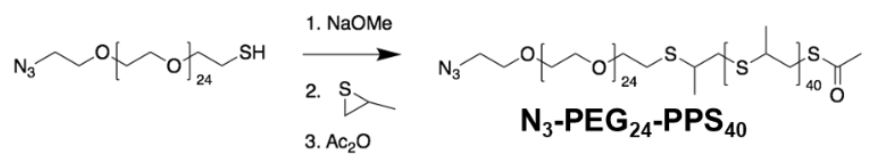

b

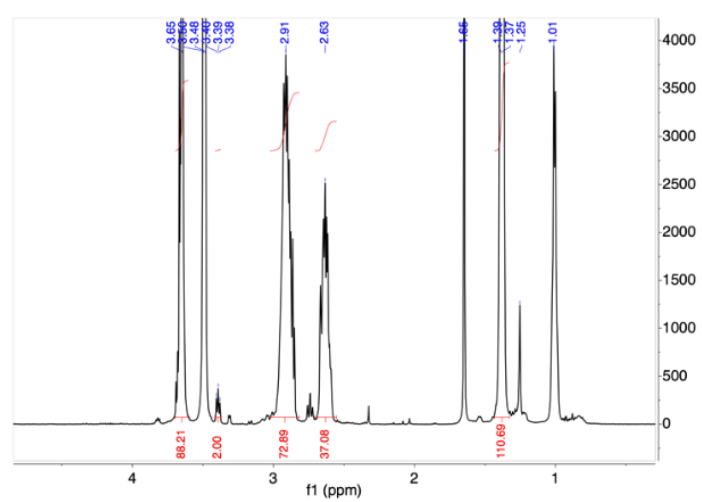

C

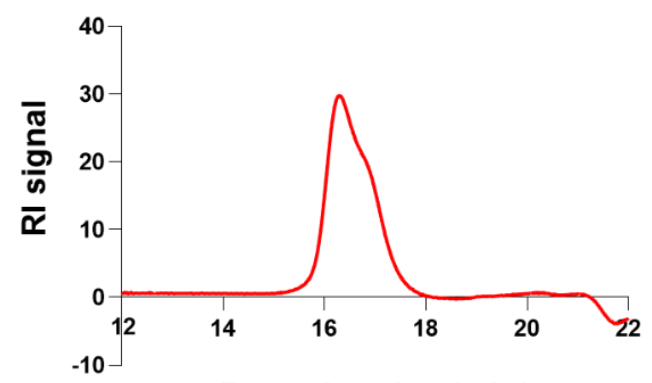

Retention time(min)

d

\begin{tabular}{|l|l|l|l|l|l|}
\hline Polymer & $\begin{array}{l}\text { DP } \\
(\text { PS) }\end{array}$ & $\begin{array}{l}\mathrm{Mn}^{\text {NMR }} \\
(\mathrm{Da})\end{array}$ & $\begin{array}{l}\mathrm{Mn}^{\mathrm{GPC}} \\
(\mathrm{Da})\end{array}$ & $\begin{array}{l}\mathrm{Mw}^{\mathrm{GPC}} \\
(\mathrm{Da})\end{array}$ & PDI \\
\hline N3-PEG-PPS & 37 & 3738 & 4030 & 4525 & 1.104 \\
\hline
\end{tabular}

Supplementary Figure S1 | Synthesis and characterization of $\mathbf{N}_{3}$-PEG-PPS. a, Synthetic route, $\mathbf{b},{ }^{1} \mathrm{H}$ NMR spectrum, $\mathbf{c}$, gel permeation chromatography (GPC) trace, and $\mathbf{d}$, summary of physiochemical properties of $\mathrm{N}_{3}$-PEG-PPS.

a

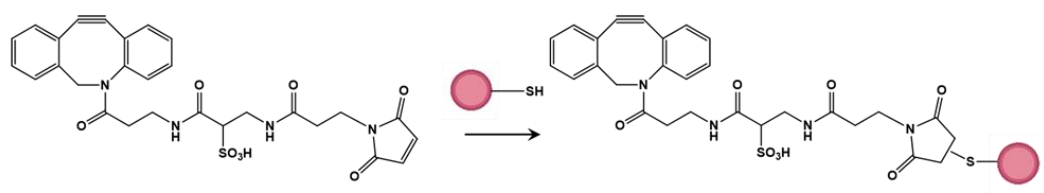

b

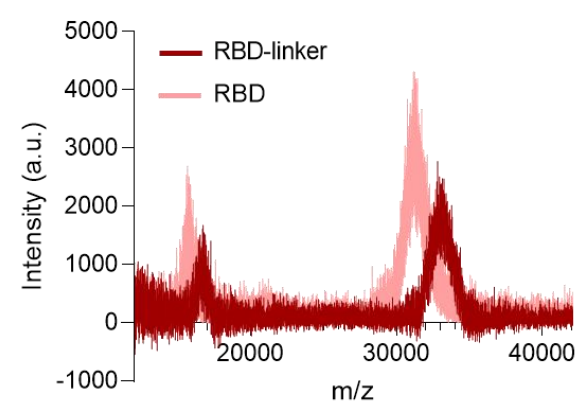

C

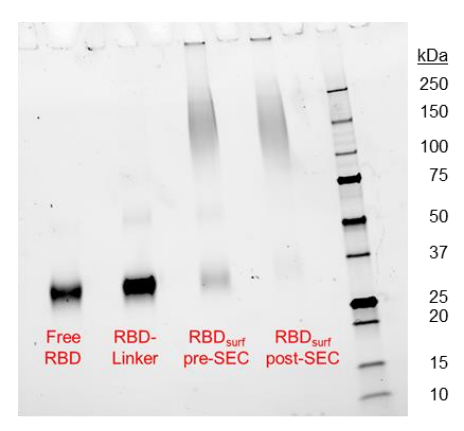

Supplementary Figure S2 | Synthesis and characterization of RBD-linker. a, Synthetic route, b, MALDI of RBD-linker and free RBD, c, SDS PAGE of free RBD, RBD-linker, RBD surf before size exclusion chromatography (SEC) and purified $\mathrm{RBD}_{\text {surt }}$ post-SEC. 
a

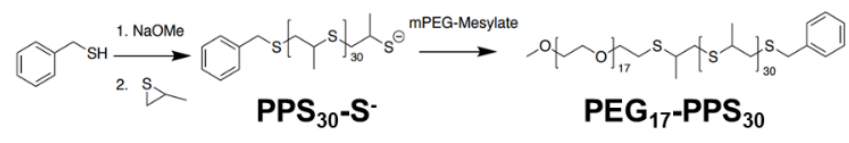

b

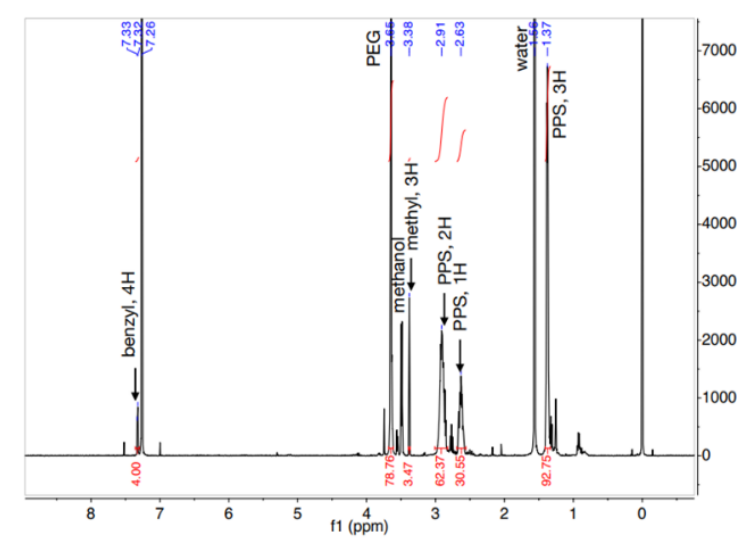

C

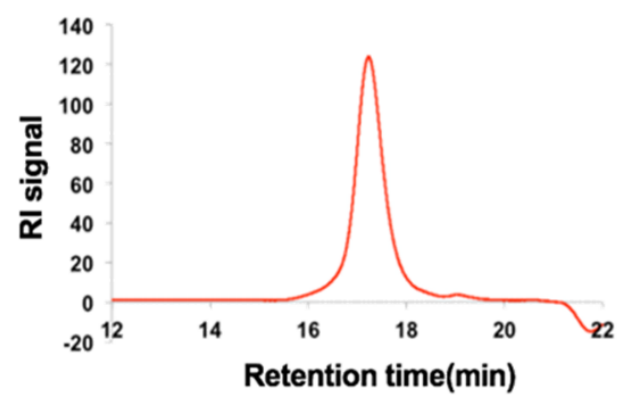

d

\begin{tabular}{|l|l|l|l|l|l|}
\hline Polymer & $\begin{array}{l}\text { DP } \\
\text { (PS) }\end{array}$ & $\begin{array}{l}\text { MnNMR } \\
\text { (Da) }\end{array}$ & $\begin{array}{l}\text { MnGPC } \\
(\mathbf{D a})\end{array}$ & $\begin{array}{l}\text { MwGPC } \\
(\text { Da) }\end{array}$ & PDI \\
\hline mPEG-PPS & 30 & 3101 & 2098 & 2337 & 1.114 \\
\hline
\end{tabular}

Supplementary Figure S3 | Synthesis and characterization of PEG-PPS. a, Synthetic route, b, ${ }^{1} \mathrm{H}$ NMR spectrum, c, gel permeation chromatography (GPC) trace, and d, summary of physiochemical properties of PEG-PPS.
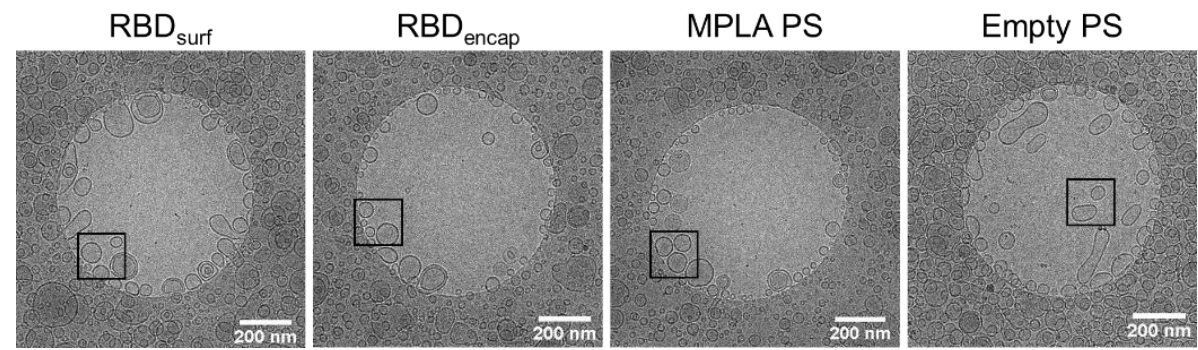

Supplementary Figure S4 | Additional cryoEM images of PS. Black box indicates magnified region in Figure 1b. 

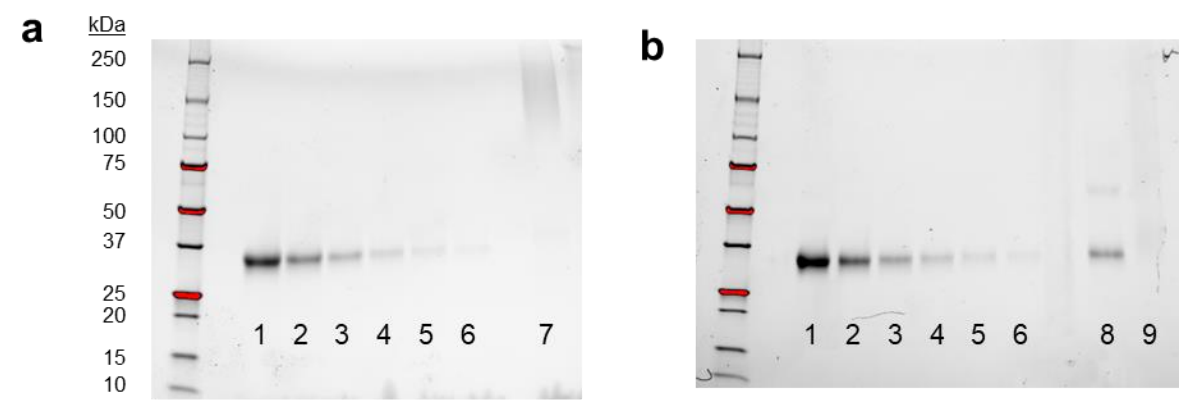

Supplementary Figure S5 | PS stability by SDS PAGE after $>180 \mathrm{~d}$ at $4^{\circ} \mathrm{C}$. SDS PAGE of a, $\mathrm{RBD}_{\text {surf }}$ and $\mathbf{b}, \mathrm{RBD}_{\text {encap. }}$. Lanes 1-6 represent RBD standard curve values of $400,200,100,50$, 25 , and $12.5 \mu \mathrm{g} / \mathrm{mL}$. Lane 7 contains of $\mathrm{RBD}_{\text {surf }}$ disrupted with Triton X. Lane 8 contains of $\mathrm{RBD}_{\text {encap }}$ disrupted with Triton $\mathrm{X}$, and Lane 9 contains undisrupted $\mathrm{RBD}_{\text {encap. }}$
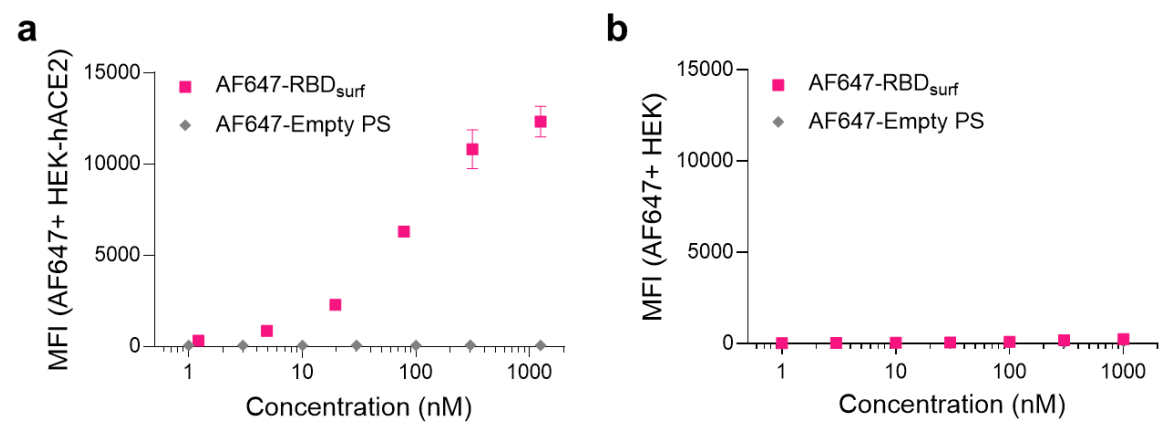

Supplementary Figure S6 | RBD binding to HEK-hACE2 and HEK-293 cells. a, Mean fluorescence intensity (MFI) of AF647-labeled RBD surf and empty PS bound to HEK-hACE2 cells characterized by flow cytometry. $\mathbf{b}, \mathrm{MFI}$ of AF647-labeled RBD surf $_{\text {and }}$ empty PS indicating an absence of binding to control HEK-293 (HEK) cells. Data plotted as mean \pm SD for $n$ $=2$ replicates. 


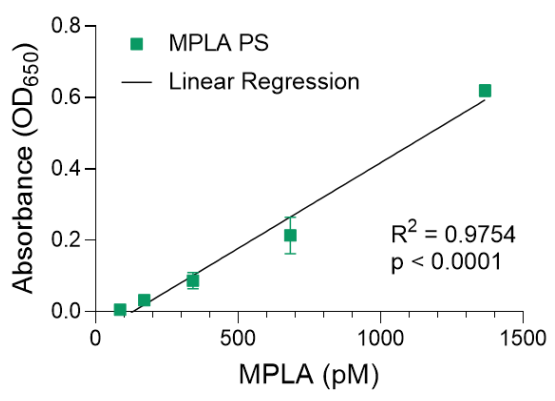

Supplementary Figure S7 | MPLA PS as a TLR4 agonist. Linear concentration-dependent stimulation of HEK-Blue ${ }^{T M}$ TLR4 reporter cells with MPLA PS. Data plotted as mean \pm SD for $n$ $=3$ replicates.
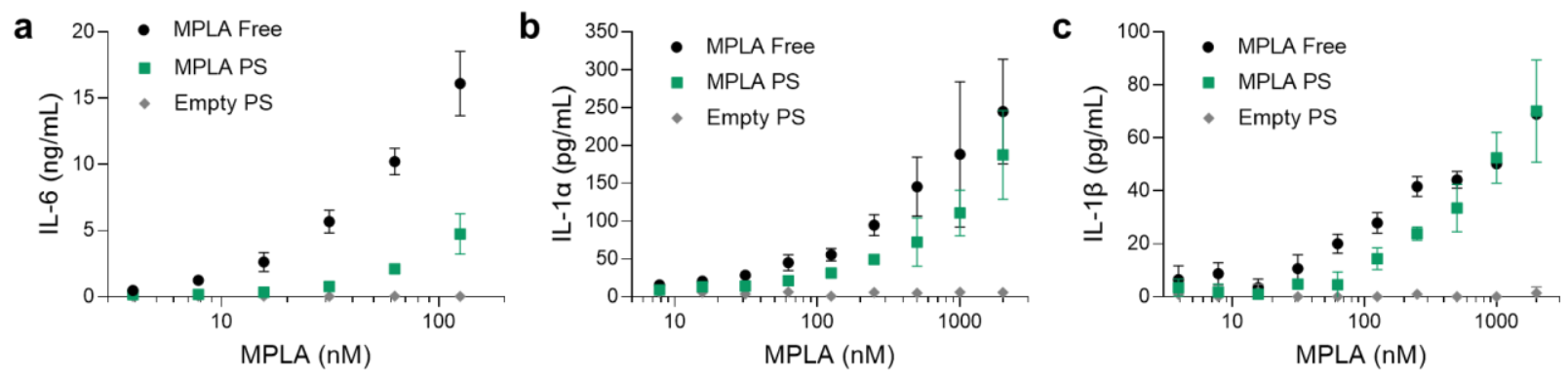

Supplementary Figure S8 | In vitro activity of MPLA PS. Dose-dependent secretion of a, IL6, b, IL-1a, and c, IL-1 $\beta$ from cultured murine bone marrow-derived dendritic cells stimulated by free MPLA, MPLA PS, or empty PS. Data plotted as mean \pm SD for $n=3$ replicates. 

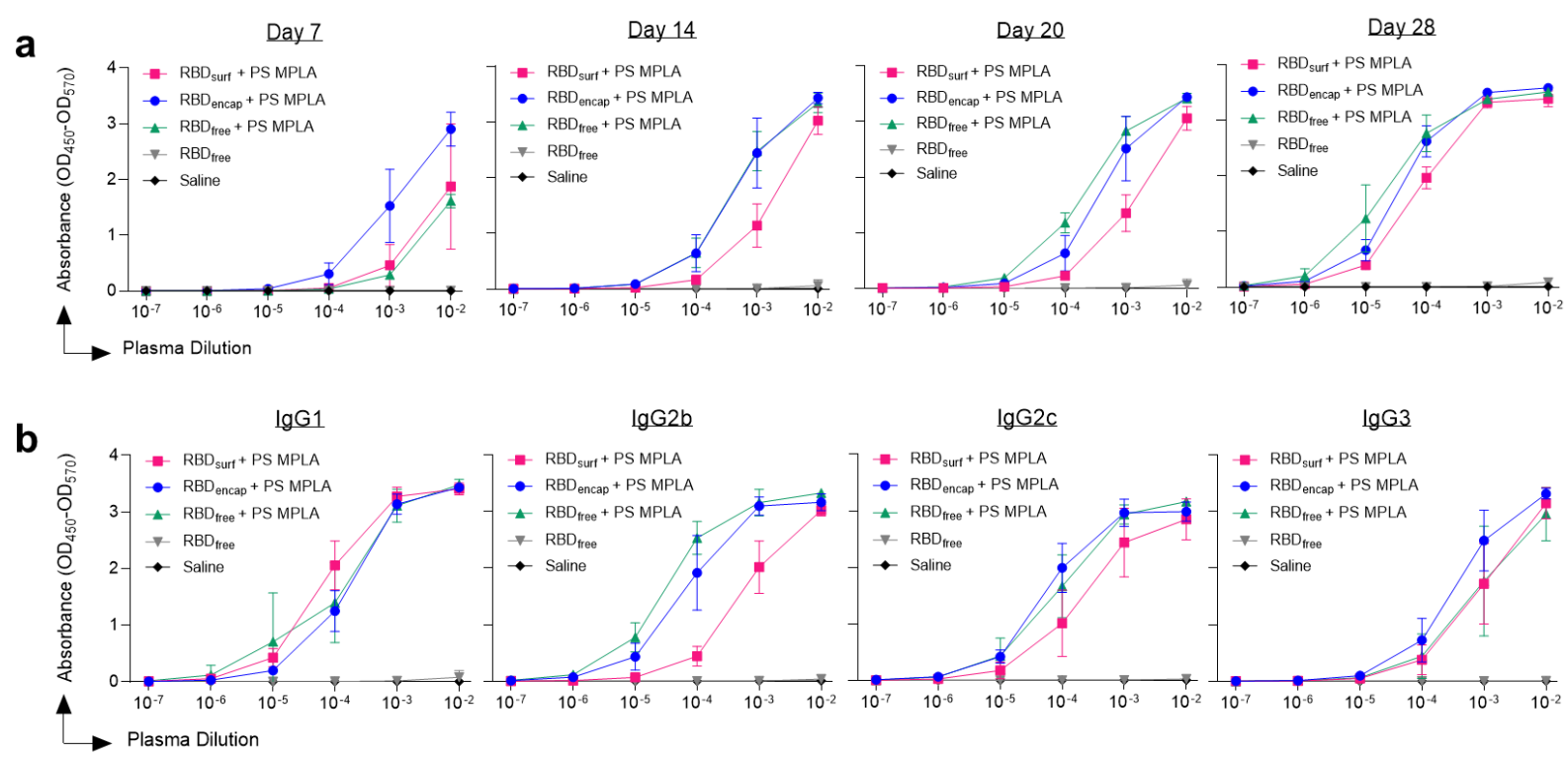

$\lg \lg 2 \mathrm{~b} G 2 \mathrm{C}$
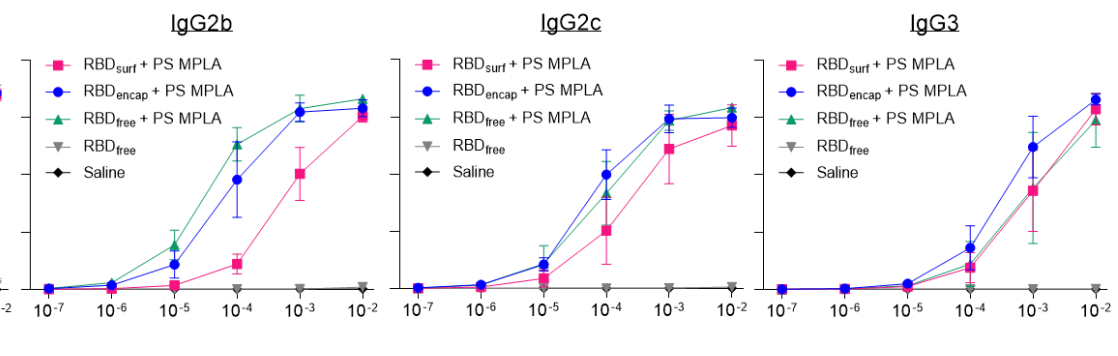

Supplementary Figure S9 | ELISA absorbance vs. dilution curves. Absorbance vs. dilution for RBD-specific ELISAs for $\mathbf{a}$, total IgG over time and $\mathbf{b}$, IgG subtypes on d28. Log-transformed curves were quantified by AUC in Figure 2. Data plotted as mean $\pm S D$ and represent 1 of 2 experiments with $n=5$ mice each.
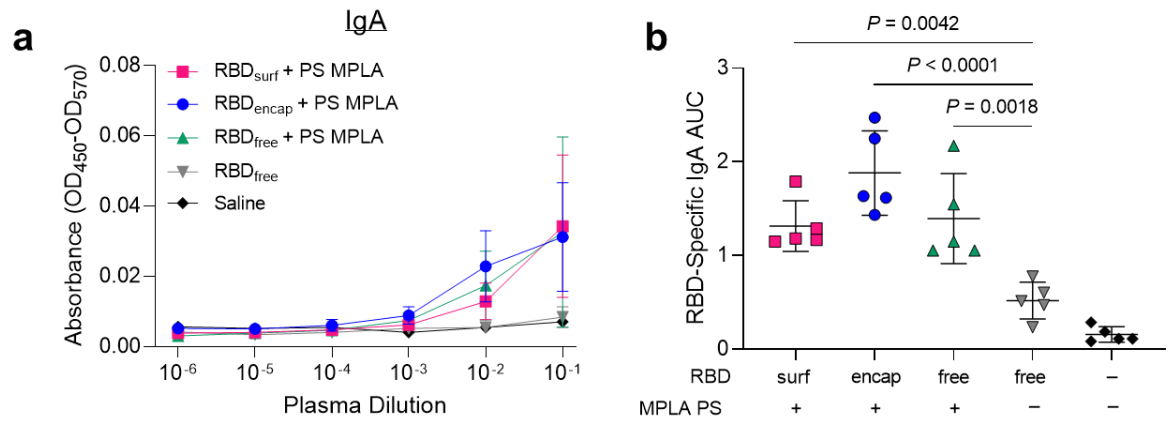

Supplementary Figure S10 | Presence of IgA antibodies. a, Absorbance vs. dilution for RBDspecific IgA ELISAs. b, AUC from (a). Data plotted as mean \pm SD and represent 1 of 2 experiments with $n=5$ mice each. Symbols in (b) represent individual mice. Comparisons to unadjuvanted $\mathrm{RBD}_{\text {free }}$ were made using one-way ANOVA with Dunn's post-test. 

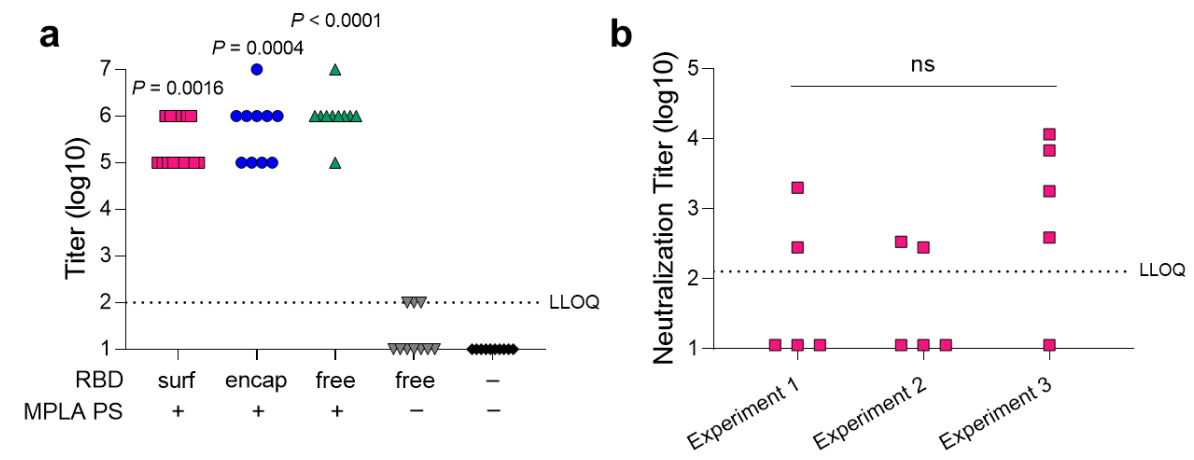

Supplementary Figure S11 | IgG antibody and viral neutralization titers. a, Aggregate RBDspecific IgG antibody titers 1 week post-boost based on ELISA. Values below the LLOQ $(=2)$ are plotted as LLOQ/2. Titers were determined as the -log of the lowest plasma dilution for which (OD450-OD570) - (average of blanks $+4 \times$ standard deviation of blanks) $>0.01$. $P$ values represent comparisons to unadjuvanted $R B D_{\text {free }}$. b) Viral neutralization titers for $R B D_{\text {surf }}+M P L A$ PS across three different cohorts of $n=5$ mice, indicating experiment reproducibility. Values below the $\operatorname{LLOQ}(=2.11)$ are plotted as LLOQ/2.; $n s p=0.11$. Symbols represent individual mice. Comparisons were made using a Kruskal-Wallis nonparametric test with Dunn's post-test.

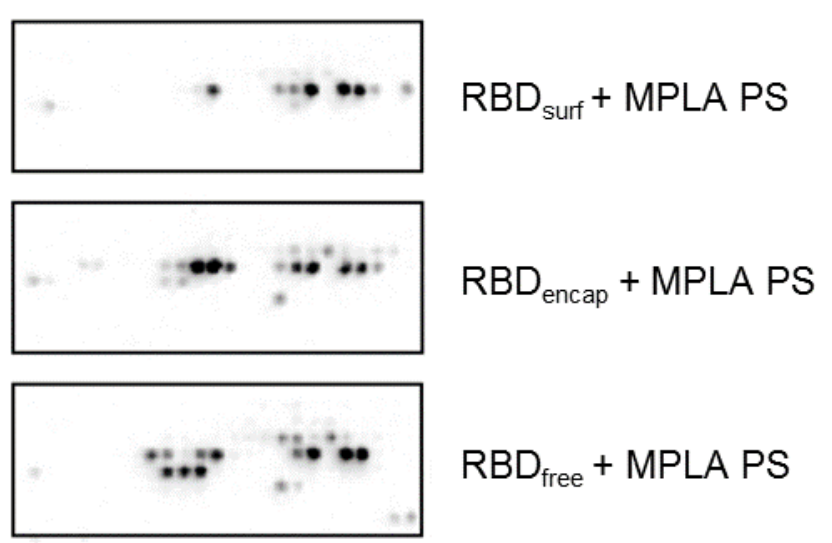

Supplementary Figure S12 | Representative peptide array images. Boxes represent region of peptide array specific to the RBD of the Spike protein. Peptide arrays quantified in Figure 3c. 
a
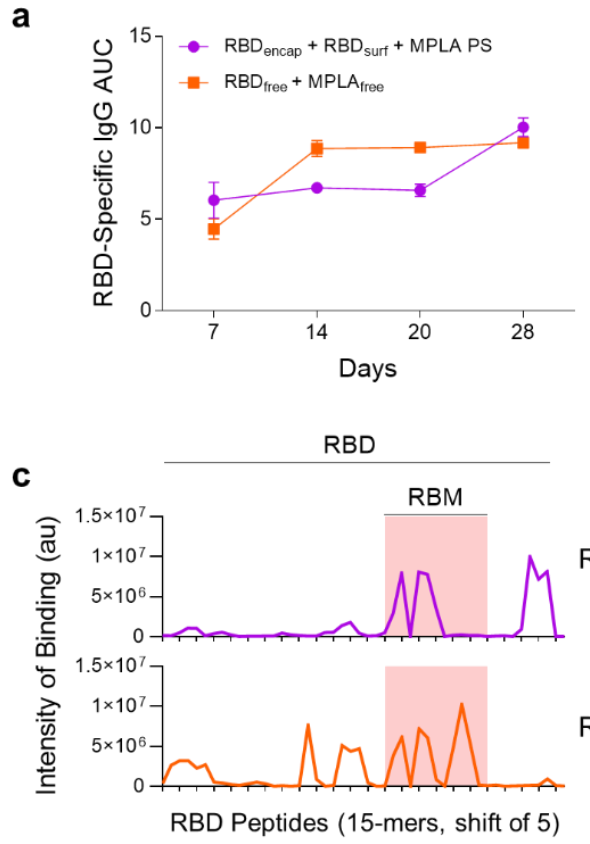

b

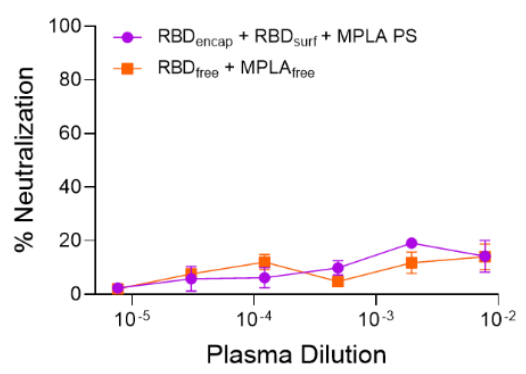

$\mathrm{RBD}_{\text {surf }}+\mathrm{RBD}_{\text {encap }}$ + MPLA PS

$\mathrm{RBD}_{\mathrm{free}}+\mathrm{MPLA}_{\mathrm{free}}$

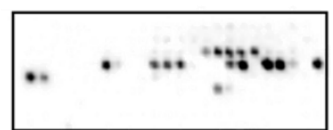

Supplementary Figure S13 | Analysis of plasma by mice vaccinated with $R B D_{\text {surf }}+R_{B D} D_{\text {encap }}$ + MPLA PS and $\mathbf{R B D}_{\text {free }}+$ MPLA free. Mice received a priming dose on day 0 with a boost on day 21 , and plasma was taken weekly to monitor production of RBD-specific antibodies. a, AUC of absorbance curve of RBD-specific IgG ELISAs for mice vaccinated with either $5 \mu \mathrm{R} R \mathrm{R}_{\text {encap }}+5$ $\mu \mathrm{g} \mathrm{RBD}_{\text {surf }}+\mathrm{MPLA} P S$ or $10 \mu \mathrm{g} \mathrm{RBD}_{\text {free }}+\mathrm{MPLA}_{\text {free }}$. Data plotted as mean $\pm \mathrm{SD}$ for $\mathrm{n}=5$ mice. b, Neutralization of SARS-CoV-2 infection of Vero E6 cells in vitro. Data plotted as mean \pm SEM for $\mathrm{n}=5$ mice. c, Epitope mapping using 15-amino-acid peptides with a 5-amino-acid shift, spanning the entire RBD sequence with representative images of peptide arrays.

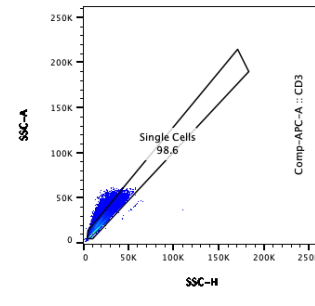

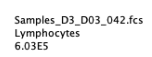

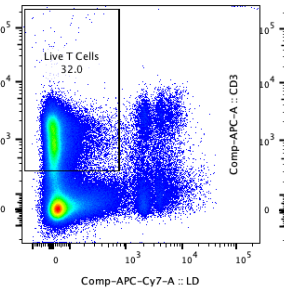

Samples_D3_D03_042.fes
Single Cellls
5.94E5

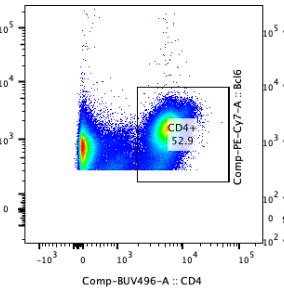

Samples_D__003__042.fcs
Live TCe-1ls
190437

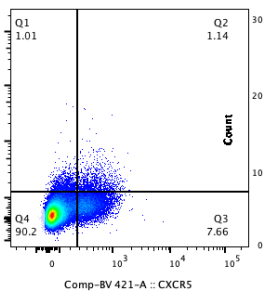

Samples__03_D03_042.tcs
CD4+_
100681

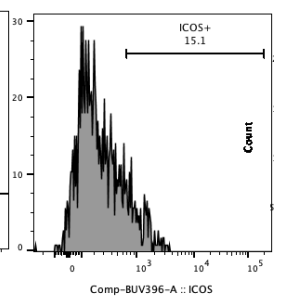

Samples.D3_D03_042.fics
O2: CCCRS+. Bc16+
1146

Supplementary Figure S14 | Representative T follicular helper cell gating strategy. 

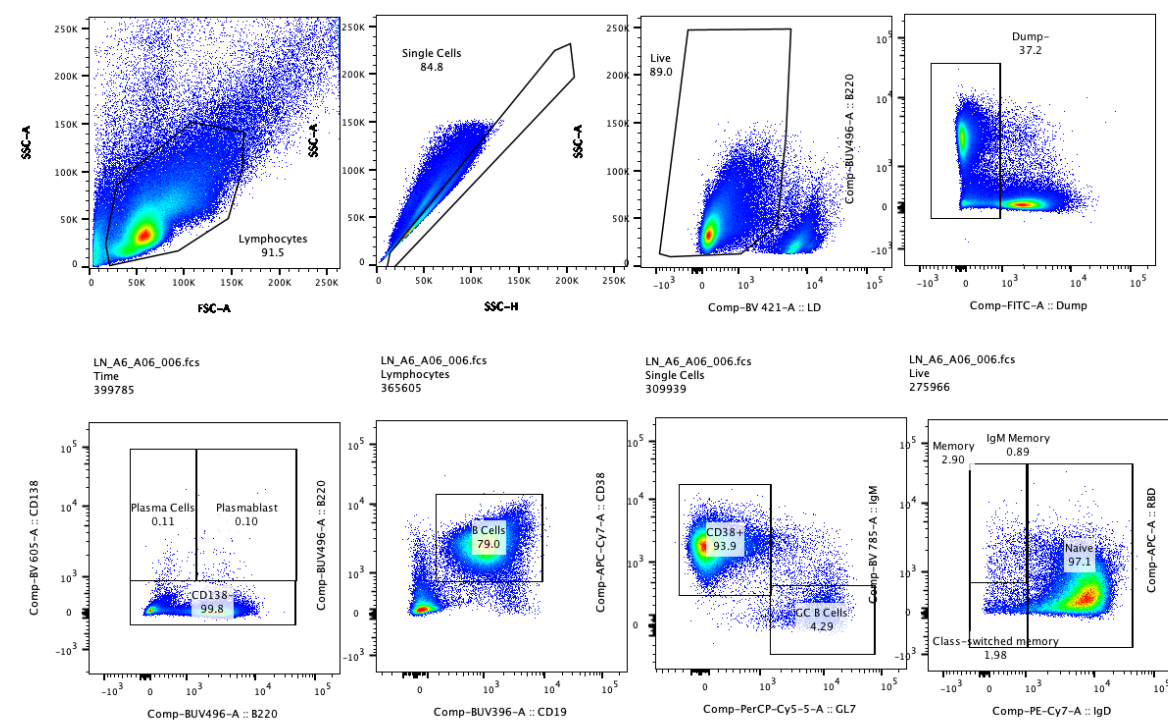

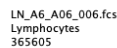

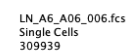

LNAG_A06__006.tcs
Live
275966
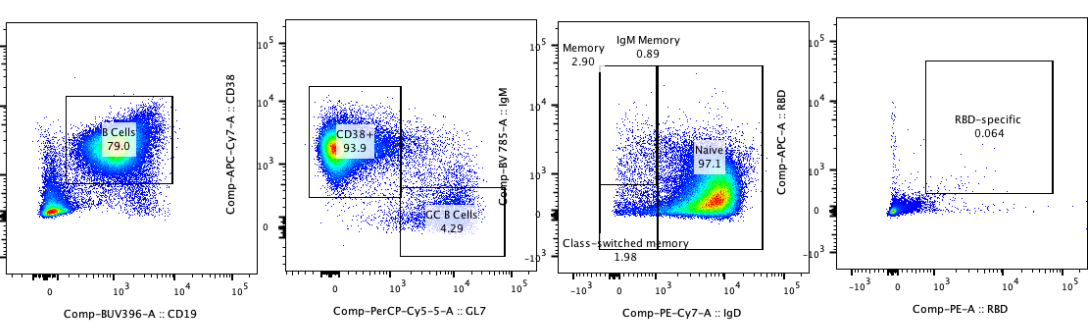

LN_A6_A06__006.fcs
Dump-
102572

LNAAGAA6_006.fes
Dump-

LNA_A6_A06_006.fCs
BCells
81025

LNAA6_A06_-006.fCs
CDS88+
76055

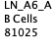

\section{Supplementary Figure S15 | Representative B cell gating strategy.}
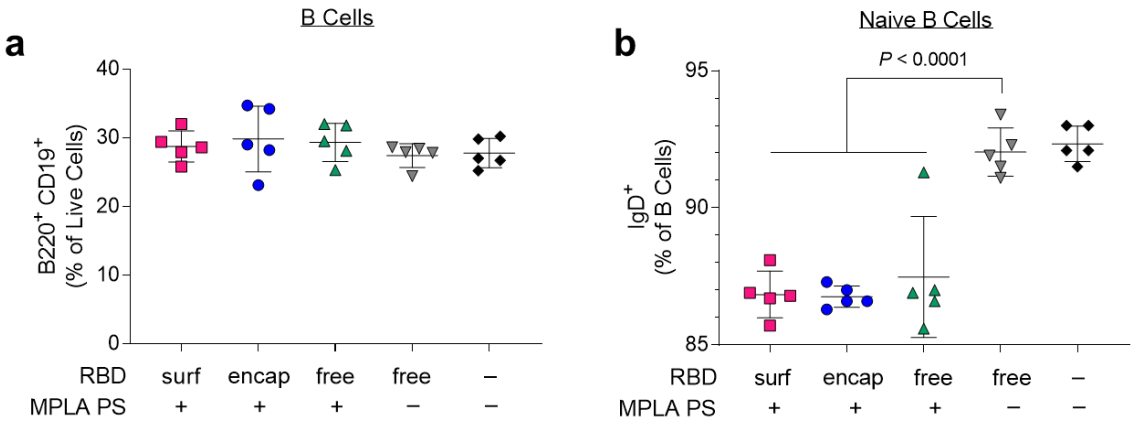

Supplementary Figure S16 | Total and naïve B cells in vaccinated mice 1 week post-boost. $\mathbf{a}$, Total B cells $\left(B 220^{+} \mathrm{CD} 19^{+}\right)$and $\mathbf{b}$, naïve B cells in dLNs. Data plotted as mean \pm SD and represent 1 of 2 experiments with $n=5$ mice each. Symbols represent individual mice. Comparisons to unadjuvanted $\mathrm{RBD}_{\text {free }}$ were made using one-way ANOVA with Dunn's post-test. 

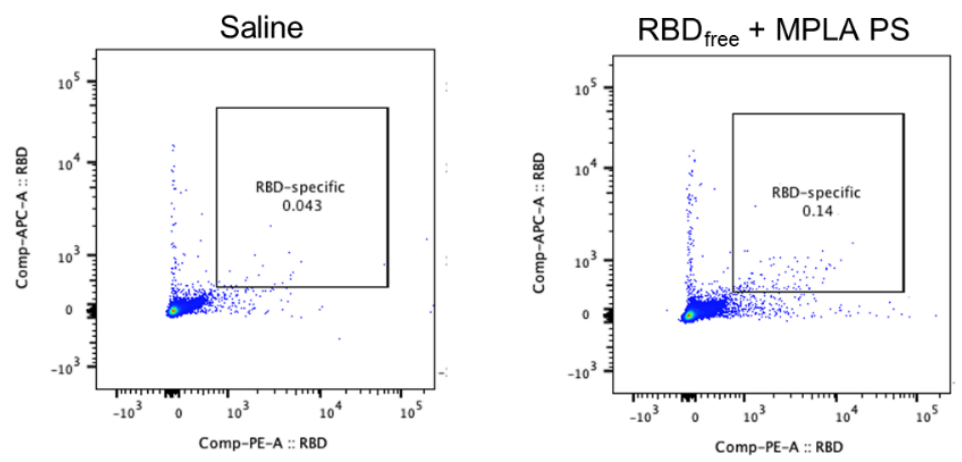

\section{Supplementary Figure S17 | Representative tetramer staining.}

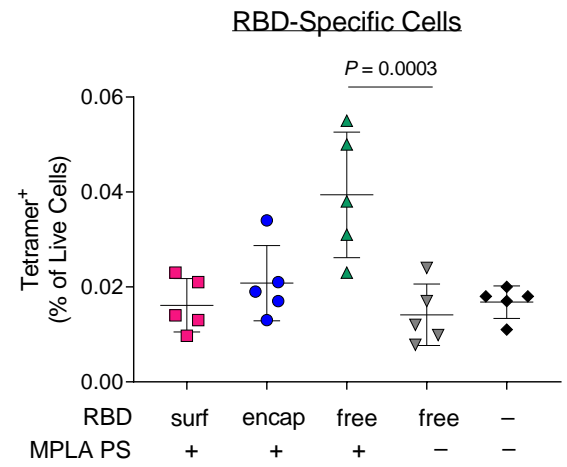

Supplementary Figure S18 | RBD-specific cells in vaccinated mice 1 week post-boost. Tetramer $^{+}$cells in dLNs on d28. Data plotted as mean \pm SD and represent 1 of 2 experiments with $n=5$ mice each. Symbols represent individual mice. Comparisons to unadjuvanted $R B D_{\text {free }}$ were made using one-way ANOVA with Dunn's post-test. 


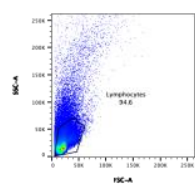

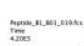

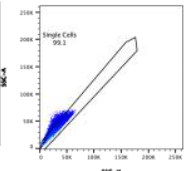

Timentions

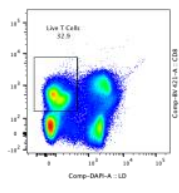

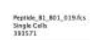

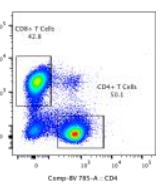

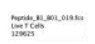
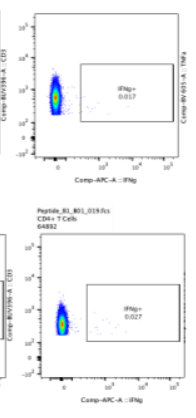

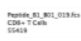
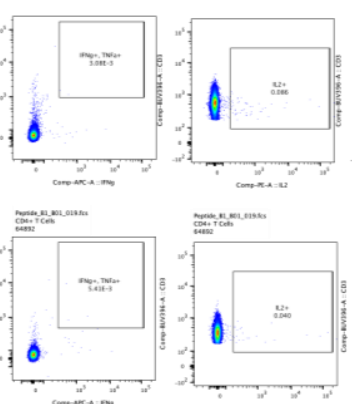

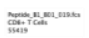

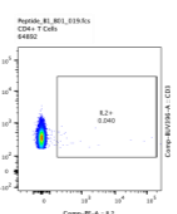

Din
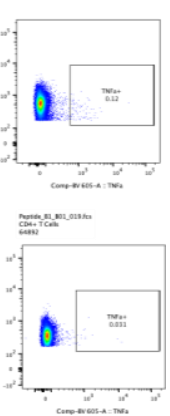

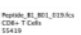

Supplementary Figure S19 | Representative intracellular cytokine gating strategy.

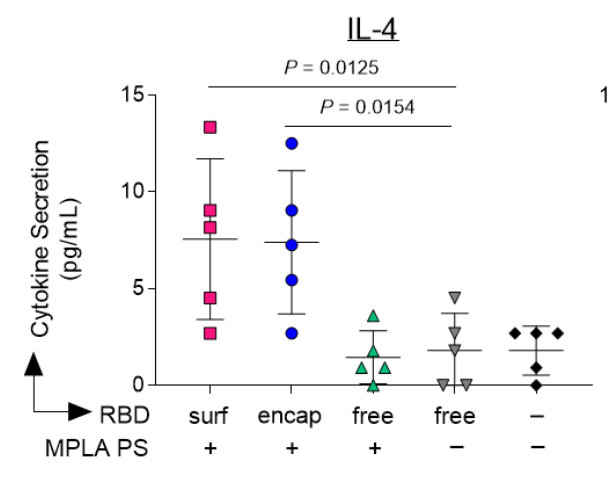

$\underline{\mathrm{IL}-5}$

$\mathrm{IL}-13$
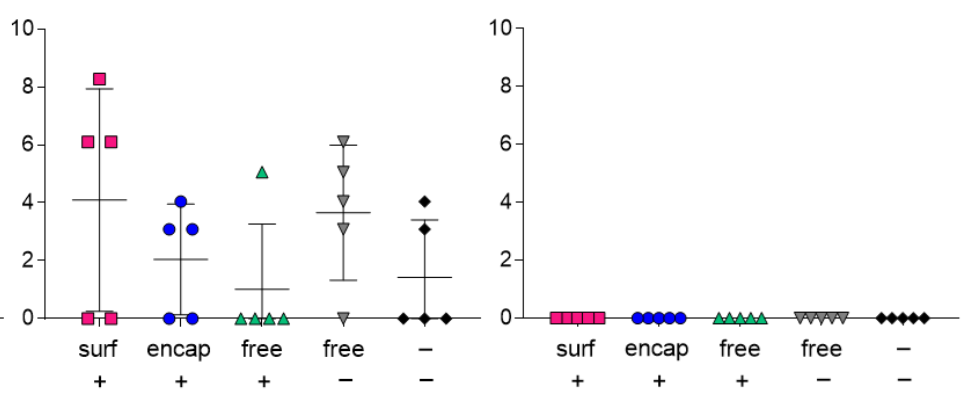

Supplementary Figure S20 | Th2-type cytokines secreted upon ex vivo stimulation with RBD. Lymph node cells isolated from the dLNs of PS vaccinated mice 1 week post-boost were restimulated ex vivo with full RBD protein. After $3 \mathrm{~d}$, levels of IL-4, IL-5, and IL-13 secreted into the supernatant were measured. Data plotted as mean \pm SD and represent 1 of 2 experiments with $n=5$ mice each. Symbols represent individual mice. Comparisons to unadjuvanted $R B D_{\text {free }}$ were made using one-way ANOVA with Dunn's post-test. 


\section{Supplementary Table S1 | Summary of loading capacities of PS.}

\begin{tabular}{|l|l|l|l|}
\hline & Polymer $\left(\mathbf{m g ~ m L}^{-1}\right)$ & Cargo $\left.\mathbf{( m g ~} \mathbf{~ m L}^{-1}\right)$ & Loading (wt\%) \\
\hline$R^{\prime} D_{\text {surf }}$ & 7.96 & 0.127 & 1.57 \\
\hline$R B D_{\text {encap }}$ & 7.00 & 0.125 & 1.75 \\
\hline MPLA PS & 3.49 & 0.241 & 6.46 \\
\hline
\end{tabular}

Polymer concentration was determined by GPC, RBD concentration in RBD surf was determined by CBQCA protein quantitation assay, RBD concentration in $\mathrm{RBD}_{\text {encap }}$ was determined by SDS PAGE, and MPLA concentration was determined by mass spectrometry.

\section{Supplementary Table S2 | Probes and antibodies for $T_{\text {fh }}$ cell panel.}

\begin{tabular}{|l|l|l|}
\hline Marker & Color & Vendor \\
\hline Viability Dye & eFluor 780 & Invitrogen 65-0865-14 \\
\hline CD4 & BV496 & BD Horizon 612952 \\
\hline CD3 & BUV737 & BD Optibuild 741788 \\
\hline CD44 & PerCpCy5.5 & Invitrogen 45-0441-82 \\
\hline CXCR5 & BV421 & Biolegend 145512 \\
\hline ICOS & BUV396 & BD Horizon 565885 \\
\hline Bcl6 & PE-Cy7 & Biolegend 358512 \\
\hline
\end{tabular}

Supplementary Table S3 | Probes and antibodies for RBD-specific B cell panel.

\begin{tabular}{|l|l|l|}
\hline Marker & Color & Vendor \\
\hline Viability Dye & Violet fluorescent reactive dye & Invitrogen L34964A \\
\hline RBD-tetramer & PE & - \\
\hline RBD-tetramer & APC & - \\
\hline F4/80 (Dump) & FITC & Biolegend 123107 \\
\hline CD11c (Dump) & FITC & Biolegend 117306 \\
\hline Ly6c(Dump) & FITC & Invitrogen 53-5932-82 \\
\hline Ly6g (Dump) & FITC & Invitrogen 11-9668-82 \\
\hline CD4 (Dump) & FITC & Biolegend 100406 \\
\hline CD8a (Dump) & FITC & Biolegend 100706 \\
\hline B220 & BUV496 & BD Horizon 612950 \\
\hline CD19 & BUV396 & BD Horizon 565965 \\
\hline CD138 & BV605 & Biolegend 142531 \\
\hline IgM & BV786 & BD Optibuild 743328 \\
\hline IgD & PE-Cy7 & Biolegend 405720 \\
\hline CD38 & APC-Cy7 & Biolegend 102727 \\
\hline GL7 & PerCP-Cy5.5 & Invitrogen 46-5902-82 \\
\hline
\end{tabular}




\section{Supplementary Table S4 | Probes and antibodies for restimulation panel.}

\begin{tabular}{|l|l|l|}
\hline Marker & Color & Vendor \\
\hline Viability Dye & eFluor 455 (UV) & Invitrogen 65-0868-14 \\
\hline CD3 & BUV395 & BD Horizon 563565 \\
\hline CD4 & BV786 & BD Horizon 563331 \\
\hline CD8 & BV421 & BD Horizon 563898 \\
\hline IFN $\lambda$ & APC & Biolegend 505810 \\
\hline TNF $\alpha$ & BV605 & Biolegend 506329 \\
\hline IL-2 & PE & BD Pharmigen 554428 \\
\hline
\end{tabular}

\section{Chemical Synthesis and Characterization}

$\mathbf{N}_{3}$-PEG-PPS. N ${ }_{3}$-PEG-PPS was synthesized by first dissolving $\mathrm{N}_{3}-\mathrm{PEG}_{24}-\mathrm{SH}$ (1 eq, $\mathrm{MW} \sim 1000 \mathrm{~g}$ $\mathrm{mol}^{-1}$; Nanosoft Polymers) in degassed, anhydrous THF and deprotonating the thiol group by addition of sodium methoxide ( $\mathrm{NaOMe} ; 1.1 \mathrm{eq}$ ) under nitrogen gas. Propylene sulfide (40 eq) was added by syringe, and the reaction proceeded until completion at the desirable degree of polymerization of PPS, as determined by ${ }^{1} \mathrm{H}$ NMR. The polymer was precipitated multiple times in ice cold methanol to obtain the final product, $\mathrm{N}_{3}-\mathrm{PEG}_{24}-\mathrm{PPS}_{40}$, characterized by ${ }^{1} \mathrm{H}$ NMR (400 $\mathrm{MHz}$ Bruker DRX spectrometer equipped with a BBO probe, using Topspin 1.3) and gel permeation chromatography (GPC; Tosoh EcoSEC size exclusion chromatography system with a Tosoh SuperAW3000 column). ${ }^{1} \mathrm{H}-\mathrm{NMR}\left(400 \mathrm{MHz}, \mathrm{CDCl}_{3}\right.$ ) of $\mathrm{N}_{3}$-PEG-PPS, $\delta 1.37$ (s, PPS, $3 \mathrm{H}), 2.63$ (m, PPS, 1H), 2.91 (m, PPS, 2H), 3.39 (t, $\left.-\mathrm{CH}_{2}-\mathrm{N}_{3}, 2 \mathrm{H}\right), 3.65$ (m, PEG).

PEG-PPS. PEG-PPS was synthesized as previously described ${ }^{1}$. Briefly, benzyl mercaptan (1 eq.) in degassed, anhydrous THF (20 mM) was deprotonated with $\mathrm{NaOMe} \mathrm{(1.1} \mathrm{eq.).} \mathrm{Under} \mathrm{nitrogen}$ protection, propylene sulfide (39 eq) was rapidly added by syringe, and the reaction proceeded until completion at the desirable degree of polymerization of PPS, as determined by ${ }^{1} \mathrm{H}$ NMR. Subsequently, mPEG $_{17}$-mesylate (synthesized in-house as previously described ${ }^{1}$ ) was added, and the mixture was allowed to react overnight. The polymer was precipitated multiple times in ice cold methanol to obtain the final product, $\mathrm{PEG}_{17}-\mathrm{PPS}_{30}$, characterized by ${ }^{1} \mathrm{H}$ NMR and GPC. ${ }^{1} \mathrm{H}-\mathrm{NMR}$ (400 MHz, CDCl 3 ) of mPEG-PPS, $\delta 1.37$ (s, PPS, 3H), 2.63 (m, PPS, 1H), 2.91 (m, PPS, $2 \mathrm{H}), 3.38\left(\mathrm{~m},-\mathrm{OCH}_{3}, 3 \mathrm{H}\right), 3.65(\mathrm{~m}, \mathrm{PEG}), 7.32(\mathrm{~m}$, benzyl, 4H).

${ }^{1}$ Scott, E. A. et al. Dendritic cell activation and T cell priming with adjuvant- and antigen-loaded oxidationsensitive polymersomes. Biomaterials 33, 6211-6219 (2012). 